\title{
ARTICLE
}

Received 10 Jun 2013 | Accepted 12 Aug 2013 | Published 10 Sep 2013 DOl: 10.1038/ncomms3429

\section{Non-invasive in vivo assessment of IDH1 mutational status in glioma}

Myriam M. Chaumeil', Peder E.Z. Larson', Hikari A.I. Yoshihara', Olivia M. Danforth', Daniel B. Vigneron?, Sarah J. Nelson ${ }^{1,2}$, Russell O. Pieper ${ }^{2,3}$, Joanna J. Phillips ${ }^{2,3,4} \&$ Sabrina M. Ronen ${ }^{1,2}$

Gain-of-function mutations of the isocitrate dehydrogenase 1 (IDH1) gene are among the most prevalent in low-grade gliomas and secondary glioblastoma. They lead to intracellular accumulation of the oncometabolite 2-hydroxyglutarate, represent an early pathogenic event and are considered a therapeutic target. Here we show, in this proof-of-concept study, that $\left[1-{ }^{13} \mathrm{C}\right] \alpha$-ketoglutarate can serve as a metabolic imaging agent for non-invasive, real-time, in vivo monitoring of mutant IDH1 activity, and can inform on IDH1 status. Using ${ }^{13} \mathrm{C}$ magnetic resonance spectroscopy in combination with dissolution dynamic nuclear polarization, the metabolic fate of hyperpolarized $\left[1^{13} \mathrm{C}\right] \alpha$-ketoglutarate is studied in isogenic glioblastoma cells that differ only in their IDH1 status. In lysates and tumours that express wild-type IDH1, only hyperpolarized $\left[1^{13} \mathrm{C}\right] \alpha$-ketoglutarate can be detected. In contrast, in cells that express mutant IDH1, hyperpolarized $\left[1-{ }^{13} \mathrm{C}\right] 2$-hydroxyglutarate is also observed, both in cell lysates and in vivo in orthotopic tumours.

\footnotetext{
${ }^{1}$ Department of Radiology and Biomedical Imaging, University of California San Francisco, 1700 4th Street, San Francisco, California 94158, USA. ${ }^{2}$ Brain Tumor Research Center, University of California San Francisco, San Francisco, California 94143, USA. ${ }^{3}$ Department of Neurological Surgery, Helen Diller Research Center, University of California San Francisco, San Francisco, California 94143, USA. 4 Department of Pathology, University of California San Francisco, San Francisco, California 94143, USA. Correspondence and requests for materials should be addressed to S.M.R. (email: sabrina.ronen@ucsf.edu).
} 
G enetic screening has determined that over $70 \%$ of lowgrade diffuse glioma tumours and up to $90 \%$ of secondary glioblastoma (GBM) cases harbour a missense point mutation in one copy of the gene encoding for the cytosolic isocitrate dehydrogenase 1 (IDH1) enzyme, most commonly at residue 132 (refs 1,2). Mutations in an equivalent residue of the mitochondrial isoform isocitrate dehydrogenase 2 have also been reported in about $20 \%$ of acute lymphoblastic leukaemia ${ }^{3}$. More recently, similar mutations have been observed in other cancers, including thyroid carcinomas (16\%), cartilaginous tumours (75\%) and intrahepatic cholangiocarcinomas $(10-23 \%)^{4}$.

The role and significance of mutant and wild-type IDH1 in cancer have been studied by several groups (see recent reviews in refs 5,6). Reports from clinical samples demonstrate that IDH1 mutations are monoallelic, with one copy of the wild-type gene and one copy of the mutant gene expressed in human tumours ${ }^{7}$. Whereas wild-type IDH1 catalyses the oxidation of isocitrate to alpha-ketoglutarate $(\alpha-K G, 2$-oxoglutarate), the mutant IDH1 isoform reduces $\alpha$-KG to 2 -hydroxyglutarate (2-HG), resulting in 2-HG levels that are 10-100 times greater than that in wild-type tissue $^{1,2}$. The interaction between wild-type and mutant enzymes is still under investigation ${ }^{8,9}$; however, it is well established that the presence of mutant IDH1 and elevated 2-HG leads to inhibition of $\alpha$-KG-dependent dioxygenases, including histone demethylases and the TET family of 5-methylcytosine hydroxylases. This, in turn, leads to genome-wide changes in histone and DNA methylation ${ }^{10,11}$. The IDH1 mutation is thus an early oncogenic event that mediates tumour development through reorganization of the methylome and transcriptome ${ }^{12,13}$. In light of its critical role in tumour pathogenesis, drugs that target mutant IDH1 are now under development ${ }^{14,15}$.

In this context, non-invasive methods for real-time monitoring of IDH1 activity and modulation of 2-HG production would be valuable for tumour characterization, patient stratification and prognosis, as well as optimization of mutant IDH1 inhibitors, and subsequent longitudinal monitoring of IDH1-targeted treatments. ${ }^{1} \mathrm{H}$ magnetic resonance spectroscopy (MRS) is a non-invasive imaging method that can probe steady-state metabolite levels. It has been used as a diagnostic and prognostic tool in brain tumour patients ${ }^{16,17}$ and is able to detect the presence of 2-HG in glioma patients in vivo ${ }^{18-21}$, in preclinical rodent models ${ }^{22,23}$ and in biopsy samples ${ }^{24,25}$. An alternative, and potentially complementary, metabolic neuroimaging approach is ${ }^{13} \mathrm{C}$ MRS. This technique informs on real-time metabolic fluxes by probing the fate of exogenous ${ }^{13} \mathrm{C}$-labelled substrates. The recent development of dissolution dynamic nuclear polarization (DNP) has allowed ${ }^{13} \mathrm{C}$-labelled compounds to be hyperpolarized, dissolved into solution and injected into the sample of interest to provide a 10,000 - to 50,000-fold increase in signal-to-noise ratio (SNR) compared with thermally polarized compound ${ }^{26} \cdot{ }^{13} \mathrm{C}$ MRS of hyperpolarized compounds can thus be applied to monitor in vivo enzymatic activities ${ }^{27}$. The conversion of hyperpolarized $\left[1-{ }^{13} \mathrm{C}\right]$ pyruvate to $\left[1-{ }^{13} \mathrm{C}\right]$ lactate has been used to detect the presence of tumour and response to treatment in several preclinical models of cancer ${ }^{27,28}$, including glioma ${ }^{29-32}$. These promising findings led to the first phase I clinical trial of this technology, which was recently completed at the University of California, San Francisco ${ }^{27,33}$. It clearly demonstrated the translational value of this approach by detecting elevated ratios of hyperpolarized lactate-to-pyruvate in tumours of prostate cancer patients. In addition to pyruvate, a number of other hyperpolarized probes have been developed to monitor other metabolic pathways, measure $\mathrm{pH}$, evaluate necrosis, assess redox potential and measure vascular flow 27 .

In this report, we demonstrate the successful use of a new DNP probe, $\left[1-{ }^{13} \mathrm{C}\right] \alpha-\mathrm{KG}$, for non-invasive ${ }^{13} \mathrm{C}$ MRS imaging of IDH1 status. We show that, following exposure to hyperpolarized $\left[1-{ }^{13} \mathrm{C}\right] \alpha-\mathrm{KG}$, we can detect the production of hyperpolarized $\left[1-{ }^{13} \mathrm{C}\right] 2-\mathrm{HG}$ in lysates and orthotopic tumours of cells engineered to express mutant IDH1 but not in lysates or tumours of their isogenic counterparts engineered to express wild-type IDH1, nor in normal contralateral brain. We conclude that $\left[1-{ }^{13} \mathrm{C}\right] \alpha-\mathrm{KG}$ is a promising agent for the in vivo interrogation of IDH1 mutational status and activity.

\section{Results}

Characterization of hyperpolarized $\alpha$-ketoglutarate. To test the hypothesis that hyperpolarized $\left[1-{ }^{13} \mathrm{C}\right] \alpha-\mathrm{KG}$ can serve as an imaging probe to monitor mutant IDH1 activity (Fig. 1a), we first determined its achievable polarization level and its $T_{1}$ relaxation time. $\left[1-{ }^{13} \mathrm{C}\right] \alpha-\mathrm{KG}$ was mixed with a stable free radical in a 3:1 mixture of water and glycerol. After $1 \mathrm{~h}$, a polarization level of $16 \pm 3 \%$ was achieved in solution at $37^{\circ} \mathrm{C}$ (time constant $\tau=1,381 \mathrm{~s})$. Following dissolution to an isotonic solution $(\mathrm{pH}=7.5 \pm 0.1, n=6)$, the resonances of hyperpolarized $\left[1-{ }^{13} \mathrm{C}\right]$ $\alpha-\mathrm{KG}\left(\delta_{\mathrm{Cl} 1-\alpha \mathrm{KG}}=172.6\right.$ p.p.m. $),\left[1-{ }^{13} \mathrm{C}\right] \alpha-\mathrm{KG}$ hydrate $\left(\delta_{\alpha \mathrm{KG}-\mathrm{H}}=\right.$ 180.9 p.p.m. $)$ and $\left[5^{13} \mathrm{C}\right] \alpha-\mathrm{KG} \quad\left(\delta_{\mathrm{C} 5-\alpha \mathrm{KG}}=184\right.$ p.p.m., originating from the $1.1 \%$ natural abundance ${ }^{13} \mathrm{C}$ at the $\mathrm{C} 5$ position) were detected (Fig. 1b). The in vitro $T_{1}$ values of all three resonances were determined at 3 and 11.7 Tesla (Table 1). The in vitro $T_{1}$ values of hyperpolarized $\left[1-{ }^{13} \mathrm{C}\right] 2-\mathrm{HG}$ were determined separately (Table 1). Importantly, the lifetime of hyperpolarized $\left[1-{ }^{13} \mathrm{C}\right] \alpha-\mathrm{KG}$ at 3 Tesla was sufficiently long $\left(T_{1}=52 \pm 4 \mathrm{~s}\right)$ that we expected to be able to probe $\alpha$-KG metabolism at this field strength.

Studies in cell lysates. U87 GBM cells were transduced with a lentiviral vector coding, respectively, for wild-type IDH1 (U87IDHwt cells), or for mutant and wild-type IDH1 (U87IDHmut cells; recapitulating the monoallelic expression observed in patient samples ${ }^{7}$ ). Western blot analysis confirmed elevated mutant IDH1 protein in U87IDHmut cells, and elevated wild-type IDH1 in both cell lines (Fig. 2a and Supplementary Fig. S1). A spectrophotometric assay confirmed the functionality of the expressed mutant IDH1 (Fig. 2b); the rate of NADPH consumption was almost six times higher in U87IDHmut than that in U87IDHwt cells: $R^{\text {U87IDHmut }}=0.64 \pm 0.06$ versus $R^{\mathrm{U} 87 \mathrm{IDHwt}}=0.13 \pm 0.06 \mathrm{fmol} \mathrm{NADPH}$ consumed per minute per cell (unpaired Student's $t$-test: $P<0.01)$. Finally, one-dimensional (1D) ${ }^{1} \mathrm{H}$ MRS (Fig. 2c) confirmed the presence of $2-\mathrm{HG}$ in U87IDHmut cell lysates, with two-dimensional (2D) ${ }^{1} \mathrm{H}$ MRS of the same lysates confirming the 2-HG assignment (Fig. 2d). The level of $2-\mathrm{HG}$ U87IDHmut cells was $5.3 \pm 1.3 \mathrm{fmol} \mathrm{cell}^{-1}$ or $10.1 \pm 2.5 \mu \mathrm{molg}^{-1}$ assuming a cell diameter of $\sim 10 \mu \mathrm{m}$. 2-HG resonances were below detection level in wild-type cells.

To show that the conversion of $\alpha-\mathrm{KG}$ to $2-\mathrm{HG}$ can be detected within the hyperpolarization lifetime, we first probed the fate of hyperpolarized $\left[1-{ }^{13} \mathrm{C}\right] \alpha-\mathrm{KG}$ in cell lysates. Following injection, a new resonance was detected at 183.9 p.p.m. in U87IDHmut but not in U87IDHwt cell extracts (Fig. 3a). This resonance was assigned to $\left[1-{ }^{13} \mathrm{C}\right] 2-\mathrm{HG}$ and the assignment was further confirmed by $2 \mathrm{D}{ }^{1} \mathrm{H}-{ }^{13} \mathrm{C}$ MRS (Fig. 3b). Normalization to the $\left[5-{ }^{13} \mathrm{C}\right] \alpha$-KG peak (the $T_{1}$ of which is comparable, see Table 1) indicated that 2-HG was accumulating over time (Fig. 3c; the resonance of $\left[5-{ }^{13} \mathrm{C}\right] \alpha$-KG decreases not only because of $T_{1}$ relaxation but also because of conversion of $\alpha-\mathrm{KG}$ to $2-\mathrm{HG}$ leading to an $\sim 2 \%$ overestimate of 2 -HG production).

Studies in orthotopic tumours. Next, it was necessary to confirm that enough hyperpolarized $\left[1-{ }^{13} \mathrm{C}\right] \alpha-\mathrm{KG}$ is delivered and metabolized to produce detectable amounts of $\left[1-{ }^{13} \mathrm{C}\right] 2-\mathrm{HG}$ 

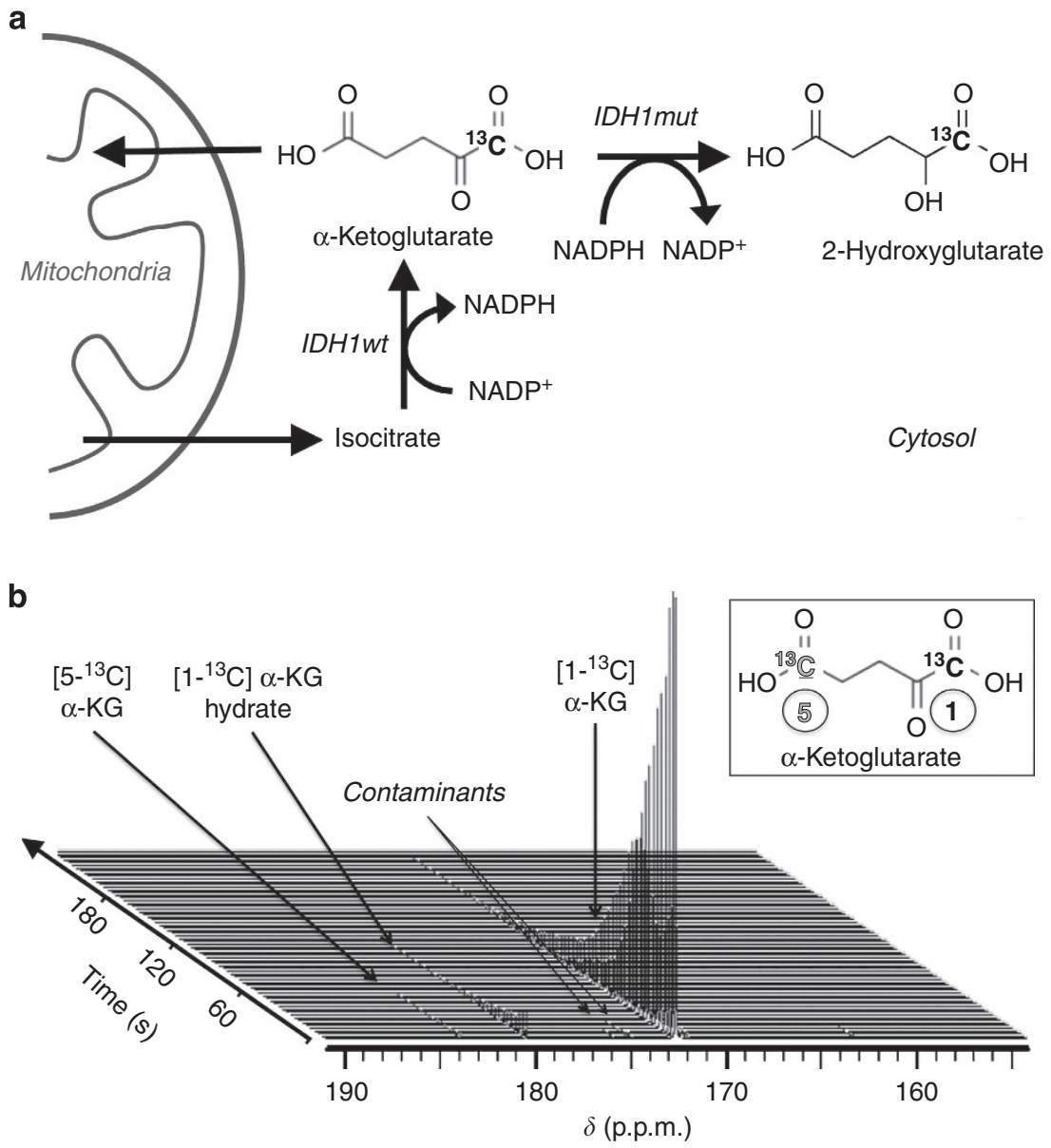

Figure $1 \mid\left[1^{-13} \mathbf{C}\right] \boldsymbol{\alpha}$-KG is a biologically relevant DNP probe. (a) Schematic of reactions catalyzed by wild-type IDH1 (IDH1wt) and mutant IDH1 (IDH1mut), and associated $\left[1-{ }^{13} \mathrm{C}\right] \alpha-\mathrm{KG}$ metabolism. The ${ }^{13} \mathrm{C}$ label at the $\mathrm{C} 1$ position of $\alpha-\mathrm{KG}$ is highlighted in bold. (b) Stack plot of ${ }^{13} \mathrm{C}$ MR spectra of hyperpolarized $\left[1{ }^{13} \mathrm{C}\right] \alpha-K G$ in solution acquired at 11.7 Tesla and showing decay of the hyperpolarized signals (temporal resolution $3 \mathrm{~s}$, polarization level $17 \%)$. Hyperpolarized $\left[1-{ }^{13} \mathrm{C}\right] \alpha-\mathrm{KG}\left(\delta_{\mathrm{Cl}-\alpha \mathrm{KG}}=172.6\right.$ p.p.m., inset black), hyperpolarized $\left[1-{ }^{13} \mathrm{C}\right] \alpha-\mathrm{KG}$ hydrate $\left(\delta_{\alpha \mathrm{KG}-\mathrm{H}}=180.9\right.$ p.p.m. $)$ and hyperpolarized $\left[5^{-13} \mathrm{C}\right] \alpha-\mathrm{KG}\left(\delta_{\mathrm{C} 5-\alpha \mathrm{KG}}=184\right.$ p.p.m., inset grey $)$ are detectable.

\section{Table 1 | $T_{1}$ relaxation times of ${ }^{13} \mathrm{C}$-labelled compounds of} interest.

\begin{tabular}{lcc} 
& \multicolumn{2}{c}{ Relaxation time $\boldsymbol{T}_{\mathbf{1}} \mathbf{( s )}$} \\
\cline { 2 - 3 } & $\mathbf{3}$ Tesla & 11.7 Tesla \\
\hline$\left[1--^{13} \mathrm{C}\right] \alpha$-Ketoglutarate & $52 \pm 4$ & $19 \pm 3$ \\
{$\left[1-{ }^{13} \mathrm{C}\right] \alpha$-Ketoglutarate hydrate } & $54 \pm 2$ & $16 \pm 4$ \\
{$\left[5--^{13} \mathrm{C}\right] \alpha$-Ketoglutarate } & $41 \pm 2$ & $18 \pm 4$ \\
{$\left[1-{ }^{13} \mathrm{C}\right]$ 2-Hydroxyglutarate } & $26^{\star}$ & $17^{\star}$ \\
\hline
\end{tabular}

In vitro relaxation times measured in solution at 3 and 11.7 Tesla $\left(37^{\circ} \mathrm{C}, \mathrm{pH}=7.5 \pm 0.1, n=3\right.$ per measurement, except for $2-\mathrm{HG}:{ }^{*} n=1$ ).

in vivo, where $\left[1-{ }^{13} \mathrm{C}\right] \alpha-\mathrm{KG}$ needs to be delivered to the brain, cross the blood-brain barrier (BBB), penetrate the tumour and permeate the cell membrane. To this end, orthotopic tumours were implanted in the right putamen of athymic rats ${ }^{31}$. When tumours reached $0.38 \pm 0.15 \mathrm{~cm}^{3}$, hyperpolarized $\left[1-{ }^{13} \mathrm{C}\right] \alpha-\mathrm{KG}$ $(V=2 \mathrm{ml}, c=100 \mathrm{mM})$ was injected intravenously.

In a first experiment, dynamic ${ }^{13} \mathrm{C}$ spectra were acquired every $3 \mathrm{~s}$ from an axial slab including the whole tumour $(2 \mathrm{~cm}$ thickness, Fig. 4a). Data were acquired from the beginning of the intravenous injection using a multi-band variable flip angle (VFA) excitation scheme optimized to monitor $2-\mathrm{HG}$ production by preserving the $\alpha$-KG hyperpolarization and efficiently using the available magnetization to enhance the detection of 2$\mathrm{HG}^{34,35}$. Hyperpolarized $\left[1-{ }^{13} \mathrm{C}\right] \alpha-\mathrm{KG}$, hyperpolarized $\left[1-{ }^{13} \mathrm{C}\right]$ $\alpha-\mathrm{KG}$ hydrate and a resonance at 184 p.p.m. were detected (Fig. 4b). The amount of $\left[1-{ }^{13} \mathrm{C}\right] \alpha-\mathrm{KG}$ in the slab was not significantly different between U87IDHwt and U87IDHmut animals, suggesting comparable delivery of the hyperpolarized substrate to the brain (Fig. 4 c). Given the $\approx 1$-p.p.m. linewidth of the 184-p.p.m. resonance in vivo, and in light of our findings in cell extracts showing that $\left[5^{13} \mathrm{C}\right] \alpha-\mathrm{KG}$ and $\left[1-{ }^{13} \mathrm{C}\right] 2-\mathrm{HG}$ are only 0.1 p.p.m. apart, these two resonances could not be spectrally resolved. Nonetheless, we found a substantial difference in the temporal evolution of the 184 p.p.m. peak between mutant and wild-type IDH1 animals. In U87IDHwt animals, this resonance presents a unimodal temporal evolution centred around the time of maximum hyperpolarized $\left[1-{ }^{13} \mathrm{C}\right] \alpha-\mathrm{KG}(\sim 10 \mathrm{~s}$ post injection) and probably corresponds to the hyperpolarized $\left[5^{-13} \mathrm{C}\right] \alpha-\mathrm{KG}$ resonance originating from the injected solution (Fig. 4d). In contrast, the same resonance follows a bimodal temporal evolution in U87IDHmut animals, suggesting a delayed metabolic production of hyperpolarized $\left[1-{ }^{13} \mathrm{C}\right] 2-\mathrm{HG}$, with a maximum around $20 \mathrm{~s}$ (Fig. 4d).

We then examined the spatial distribution of hyperpolarized metabolites using a dedicated $2 \mathrm{D}{ }^{13} \mathrm{C}$ chemical shift imaging

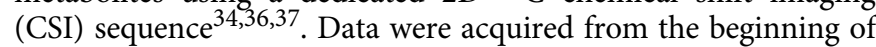


a

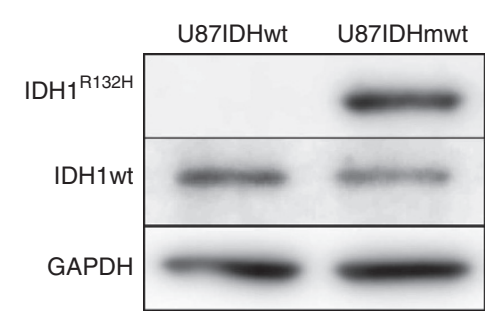

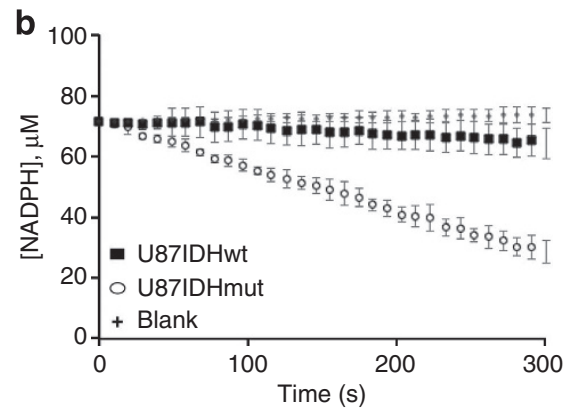

C

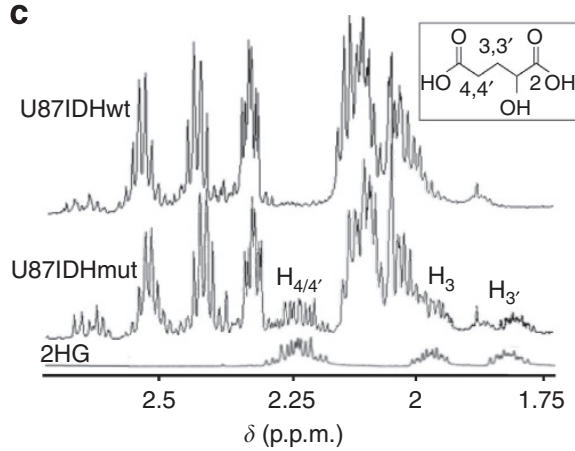

d

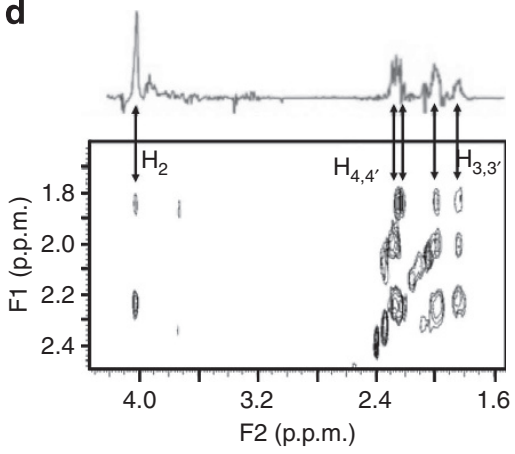

Figure 2 | Mutant IDH1 cells exhibit elevated enzyme activity and 2-HG levels. (a) Western blotting for wild-type IDH1 (IDH1wt, 46 kDa) and mutant IDH1 (IDH1 ${ }^{\mathrm{R} 132 \mathrm{H}}, 46 \mathrm{kDa}$ ) isoforms in U87IDHmut and U87IDHwt cells (GAPDH (40.2 kDa) was used as a loading control; see Supplementary Fig. S1 for full blots). (b) Spectrophotometric assay of mutant IDH1 activity in U87IDHwt (filled squares) and U87IDHmut (open circles) cells, monitoring changes in NADPH absorption over time at $340 \mathrm{~nm}$ compared with buffer blank (plus sign) (error bars: \pm s.d.m.). (c) ${ }^{1} \mathrm{H}$ spectra of pure 2-HG (bottom), U87IDHwt cell lysate (top) and U87IDHmut cell lysate (middle). Inset shows the structure of 2-HG and annotated protons detected in the spectrum. (d) Zoomed view of $2 \mathrm{D}{ }^{1} \mathrm{H}$ total correlation spectroscopy of a U87IDHmut cell lysate acquired at 14 Tesla, illustrating the cross peaks characteristic of 2-HG $\left(\mathrm{H}_{2}=4.00\right.$ p.p.m.; $\mathrm{H}_{3,3^{\prime}}=1.85 / 2.00$ p.p.m.; $\mathrm{H}_{4,4^{\prime}}=2.25$ p.p.m.). A projection representing the sum of all slices containing the $2-\mathrm{HG}$ spin system is shown on top of the total correlation spectroscopy box.

the intravenous injection every $5 \mathrm{~s}$ with an in-plane spatial resolution of $5 \mathrm{~mm}$. A multi-band excitation scheme was used to increase the signal of $2-\mathrm{HG}$ by preserving the magnetization of its precursor $\alpha-\mathrm{KG}^{34}$.

The grid used for 2D CSI acquisition is shown as an overlay to a T2-weighted MR image for a U87IDHmut animal (Fig. 5a). Tumour volumes were not significantly different between U87IDHmut and U87IDHwt tumour-bearing animals $\left(V^{\mathrm{wt}}=0.38 \pm 0.19 \mathrm{~cm}^{3}\right.$ versus $V^{\mathrm{mut}}=0.38 \pm 0.12 \mathrm{~cm}^{3}$, unpaired Student's $t$-test: $P=0.7)$. The levels of hyperpolarized $\left[1-{ }^{13} \mathrm{C}\right] \alpha$ KG were measured in tumour voxels (red/blue, $n=6$, including $n=3$ U87IDHmut and $n=3$ U87IDHwt), in normal brain voxels (green, $n=6$ ) and in blood-containing voxels centred on the basal artery (orange, $n=6$ ) for each imaging time point (Fig. $5 \mathrm{~b}$ ). As expected, the levels of hyperpolarized $\left[1-{ }^{13} \mathrm{C}\right] \alpha-\mathrm{KG}$ were significantly higher in blood-containing voxels as compared with normal brain (unpaired Student's $t$-test: ${ }^{* *} P<0.005$ at $10,15,20$ and $25 \mathrm{~s} ;{ }^{\star} P<0.05$ at $\left.30 \mathrm{~s}\right)$. In addition, significantly higher levels of hyperpolarized $\left[1-{ }^{13} \mathrm{C}\right] \alpha-\mathrm{KG}$ were observed in the tumour voxels as compared with normal brain (unpaired Student's $t$-test: ${ }^{*} P<0.005$ at $10,15,20$ and $25 \mathrm{~s} ;{ }^{*} P<0.05$ at $\left.30 \mathrm{~s}\right)$ and no significant differences in hyperpolarized $\left[1-{ }^{13} \mathrm{C}\right] \alpha-\mathrm{KG}$ levels were observed between tumour and blood-containing voxels, suggesting higher delivery and retention of hyperpolarized $\left[1-{ }^{13} \mathrm{C}\right] \alpha-\mathrm{KG}$ in the tumour region. This effect is consistent with increased angiogenesis, elevated vascular permeability and BBB breakdown in the tumour ${ }^{31}$, and probably enhances our ability to detect $\alpha$-KG metabolism within the tumour region. Importantly, hyperpolarized $\left[1-{ }^{13} \mathrm{C}\right] \quad \alpha-\mathrm{KG}$ levels were not significantly different between U87IDHwt and U87IDHmut tumours at any of the time points monitored (Fig. $5 \mathrm{c}$ ), indicating that substrate delivery to tumour is independent of tumour IDH1 status, and is thus not a confounding factor in the detection of hyperpolarized $\left[1-{ }^{13} \mathrm{C}\right] 2-\mathrm{HG}$.

Hyperpolarized ${ }^{13} \mathrm{C}$ MR spectra from one U87IDHwt tumour (blue), one U87IDHmut tumour (red), one normal brain (green) and one blood voxel (orange) show the temporal evolution of hyperpolarized metabolites (Fig. 5d). The 184-p.p.m. resonance was observed only in the mutant IDH1 tumour voxel and appeared only at $20 \mathrm{~s}$ post injection (Fig. $5 \mathrm{~d}$ ). It was not observed in wild-type tumour or blood voxels, in spite of the comparable delivery of hyperpolarized $\left[1-{ }^{13} \mathrm{C}\right] \alpha-\mathrm{KG}$ in these three voxel types. On the basis of this data, we attributed the 184-p.p.m. resonance observed at $20 \mathrm{~s}$ in mutant IDH1 tumours to hyperpolarized $\left[1-{ }^{13} \mathrm{C}\right] 2-\mathrm{HG}$. When considering all the rats studied $(n=6)$, the SNR of hyperpolarized $\left[1-{ }^{13} \mathrm{C}\right] 2-\mathrm{HG}$ was $\mathrm{SNR}^{\mathrm{mut}}{ }_{\left[1-{ }^{13} \mathrm{C}\right]} 2-\mathrm{HG}=$ $1.4 \pm 0.2$ in U87IDHmut tumours, whereas the resonance of hyperpolarized $\left[1-{ }^{13} \mathrm{C}\right] 2-\mathrm{HG}$ was not detectable in any of the U87IDHwt tumours at that time point $\left(\mathrm{SNR}^{\mathrm{wt}}{ }_{\left[1-{ }^{13} \mathrm{C}\right]} 2-\mathrm{HG}=\right.$ $1.0 \pm 0.1$; unpaired Student's $t$-test: ${ }^{\star} P<0.05$, Fig. 5e). Consequently, although the SNR of hyperpolarized $\left[1-{ }^{13} \mathrm{C}\right] 2-\mathrm{HG}$ was low, our results demonstrate statistical significance in discriminating between mutant IDH1 and wild-type tumours.

These data also served to determine the in vivo effective decays of $\left[1-{ }^{13} \mathrm{C}\right] \alpha-\mathrm{KG}$ in U87IDHwt animals, in which no metabolism of $\alpha-\mathrm{KG}$ to $2-\mathrm{HG}$ was expected. The measured decays were $17.7 \pm 1.8 \mathrm{~s}$ in the vasculature and $20.6 \pm 1.7 \mathrm{~s}$ in the normal brain. As expected, these in vivo values are shorter than the in vitro measurements, as these decays include losses of polarization because of $T_{1}$, flow and potentially metabolism ${ }^{38}$.

U87IDHwt and U87IDHmut tumours of comparable size are shown in Fig. 6a. Heatmaps illustrate high levels of 

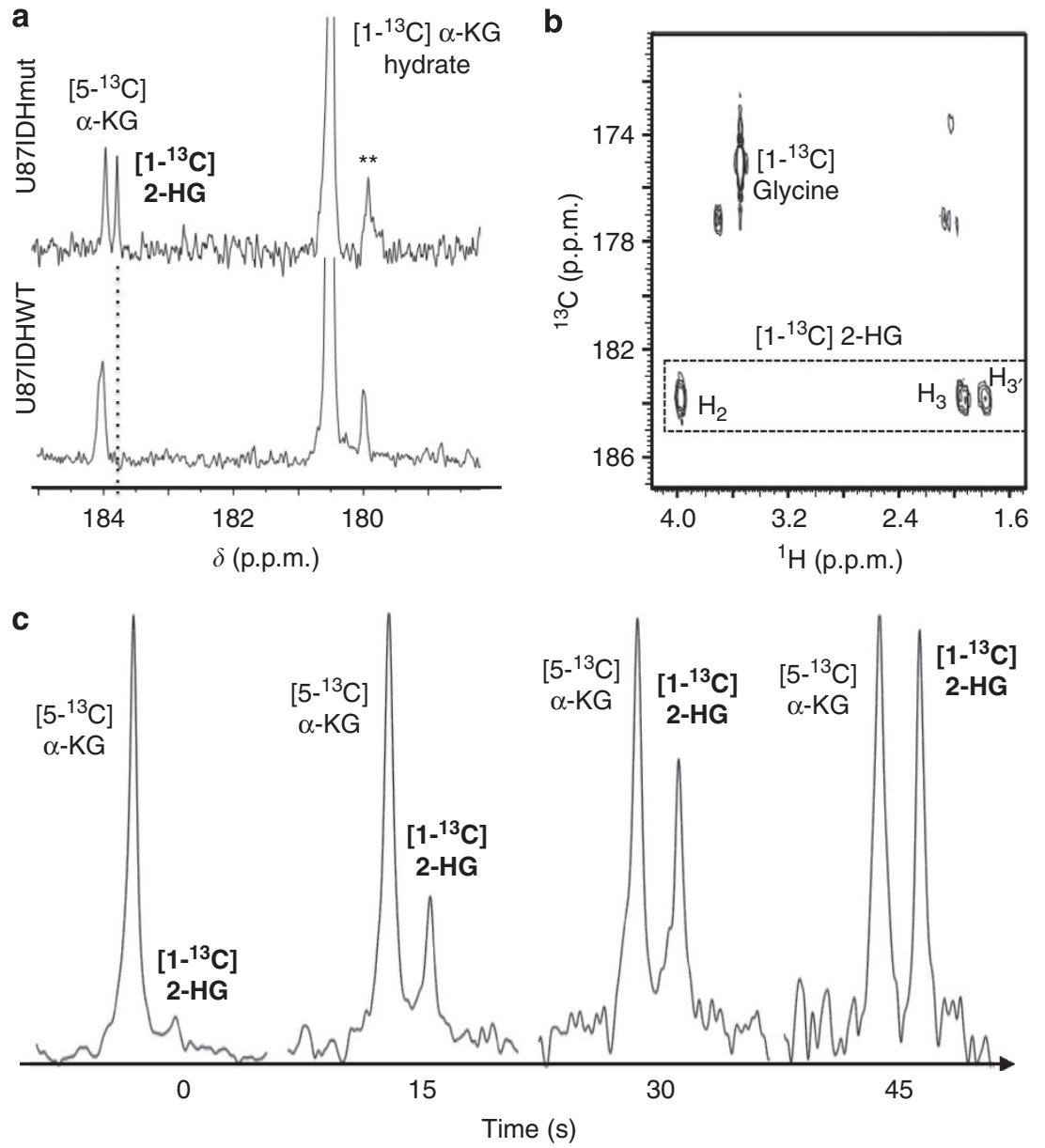

Figure 3 | Hyperpolarized 2-HG production is detected in mutant IDH1 cell lysates. (a) ${ }^{13} \mathrm{C}$ MR spectra acquired $45 \mathrm{~s}$ after injection of hyperpolarized $\left[1-{ }^{13} \mathrm{C}\right] \alpha-K G$ in U87IDHmut cell lysate (top) and U87IDHwt cell lysate (bottom; absolute intensities; ${ }^{* *}$ contaminant originating from the $\alpha$-KG preparation). (b) Gradient-selected heteronuclear multiple bond coherence spectra of the same U87IDHmut lysates acquired at 14 Tesla, confirming the production of $\left[1-{ }^{13} \mathrm{C}\right] 2-\mathrm{HG}$ from $\left[1-{ }^{13} \mathrm{C}\right] \alpha-\mathrm{KG}$. The ${ }^{1} \mathrm{H}-{ }^{13} \mathrm{C}$ cross peaks of $\left[1-{ }^{13} \mathrm{C}\right] 2-\mathrm{HG}$ (dotted box), confirm the $\left[1-{ }^{13} \mathrm{C}\right] 2-\mathrm{HG}$ assignment at $183.9 \mathrm{p} . \mathrm{p} . \mathrm{m}$. Note: $\left[1-{ }^{13} \mathrm{C}\right]$ glycine was used as an external reference. (c) Temporal evolution of $\left[1{ }^{13} \mathrm{C}\right] 2-\mathrm{HG}$ in U87IDHmut cell lysate normalized to $\left[5-{ }^{13} \mathrm{C}\right] \alpha-\mathrm{KG}$ peak intensity, showing build-up of hyperpolarized $\left[1{ }^{13} \mathrm{C}\right] 2-\mathrm{HG}$.

hyperpolarized $\left[1-{ }^{13} \mathrm{C}\right] \alpha-\mathrm{KG}$ in the blood vessels underneath the brain and in the tumours, regardless of tumour IDH1 status (Fig. 6b). In contrast, heatmaps of hyperpolarized $\left[1-{ }^{13} \mathrm{C}\right] 2-\mathrm{HG}$ show the presence of $2-\mathrm{HG}$ in U87IDHmut tumour only; this resonance is absent from blood-rich areas underneath the brain, from normal brain voxels and from U87IDHwt tumours (Fig. 6c). Similar findings were made in all U87IDHmut $(n=3)$ and U87IDHwt tumours $(n=3)$.

Post-mortem analysis. Immunohistochemical staining confirmed the presence of the mutant IDH1 isoform in U87IDHmut tumours but not in U87IDHwt (Fig. 7a). In line with these results, 2-HG was detectable using ${ }^{1} \mathrm{H}$ MRS only in the extracts of U87IDHmut tumours. The average level of 2-HG was $9.8 \pm 1.6 \mu \mathrm{molg}^{-1}$ of tumour $(n=3)$ and consistent with the estimated $10.1 \pm 2.5 \mu \mathrm{molg}^{-1}$ in cell lysates (Fig. $7 \mathrm{~b}$ ).

\section{Discussion}

Our results show that hyperpolarized $\left[1-{ }^{13} \mathrm{C}\right] \alpha-\mathrm{KG}$ fulfills the mandatory requirements for a useful $\mathrm{MR}$ metabolic imaging probe. The $T_{1}$ relaxation time of $\left[1-{ }^{13} \mathrm{C}\right] \alpha-\mathrm{KG}$ in solution at 3 Tesla $(52 \mathrm{~s})$ compared favourably with the $T_{1}$ values of other commonly used DNP probes measured at $1.5-3$ Tesla $\left(\left[1-{ }^{13} \mathrm{C}\right]\right.$ pyruvate: $T_{1} \approx 45-60 \mathrm{~s}^{39} ;\left[1-{ }^{13} \mathrm{C}\right]$ lactate: $T_{1} \approx 45 \mathrm{~s}^{40}$; and $\left[1-{ }^{13} \mathrm{C}\right]$ alanine: $\left.T_{1} \approx 40 \mathrm{~s}^{41}\right)$. The $T_{1}$ of such carbonyl-labelled DNP probes, including $\alpha-K G$, is dominated by chemical shift anisotropy (CSA) relaxation processes. As a result, although lower magnetic fields lead to poorer spectral resolution, studies of hyperpolarized $\left[1-{ }^{13} \mathrm{C}\right] \alpha-\mathrm{KG}$, like other hyperpolarized probes, benefit from lower field strengths at which the CSA is minimized (CSA- $\alpha \mathrm{B}_{0}^{2}$ ) and the $T_{1}$ is longer.

The level of liquid state polarization $\left(16 \%\right.$ at $\left.37^{\circ} \mathrm{C}\right)$ was also comparable to the values reported for other hyperpolarized ${ }^{13} \mathrm{C}$-carbonyl-labelled probes (range $7-30 \%$ ) ${ }^{27}$. Achieving this polarization level required dissolving $\left[1-{ }^{13} \mathrm{C}\right] \alpha-\mathrm{KG}$ in a glassing agent (glycerol), at high concentration $(5.9 \mathrm{M})$, and optimizing the concentrations of free radical and gadolinium agent. As our results show, the polarization level achieved using a Hypersense DNP system provided sufficient SNR for detection of hyperpolarized $\left[1-{ }^{13} \mathrm{C}\right] \alpha-\mathrm{KG}$ and its metabolic product, hyperpolarized $\left[1-{ }^{13} \mathrm{C}\right]$ $2-\mathrm{HG}$, in an animal in vivo. Further enhancements in polarization level could be achieved, thanks to newly developed equipment such as the GE SpinLab clinical polarizer, which has been shown to increase polarization levels by the use of a lower temperature and higher magnetic field for $\mathrm{DNP}^{42,43}$.

A potential limitation of our study is the model system, which was genetically engineered to express mutant IDH1. We chose 
a

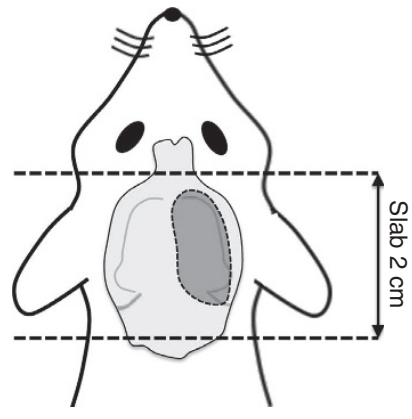

C

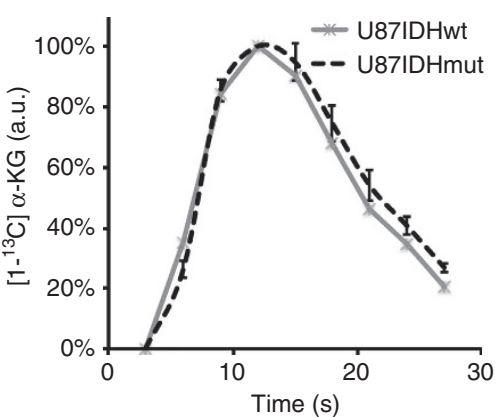

b

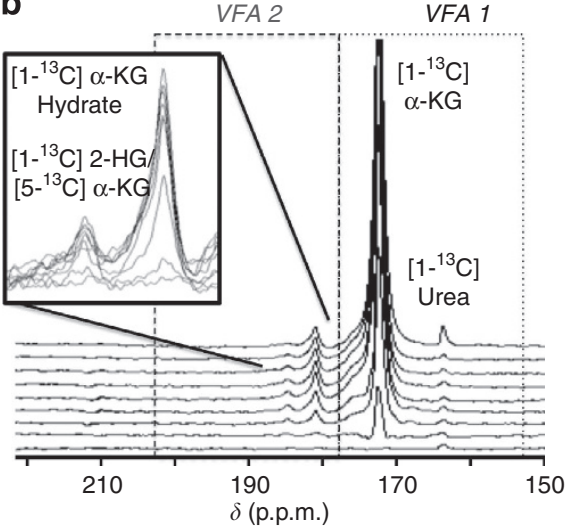

d

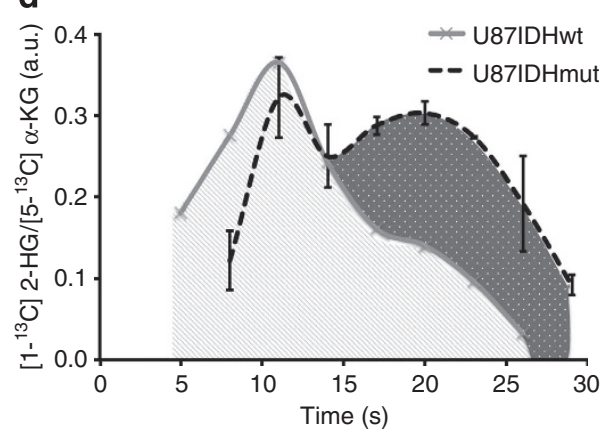

Figure 4 | Hyperpolarized 2-HG can be detected in vivo in mutant IDH1 tumours. (a) Schematic of axial slab used for in vivo dynamic ${ }^{13} \mathrm{C} M R$ experiments. The $2-\mathrm{cm}$ slab was positioned to include the whole tumour while minimizing contamination from neck and nose blood vessels. (b) Stack plot of in vivo dynamic ${ }^{13} \mathrm{C}$ MR spectra acquired from a $2-\mathrm{cm}$ slab following injection of hyperpolarized $\left[1-{ }^{13} \mathrm{C}\right] \alpha-\mathrm{KG}$ in a tumour-bearing $\mathrm{U} 87 \mathrm{IDHmut}$ rat (temporal resolution $5 \mathrm{~s} ; \mathrm{lb}=20 \mathrm{~Hz}$ ). A VFA scheme (VFA1/VFA2, see Methods) was used to improve the signal of hyperpolarized [1-13 $\mathrm{C}] 2-\mathrm{HG}$. Signals from hyperpolarized $\left[1{ }^{-13} \mathrm{C}\right] \alpha-\mathrm{KG}\left(\delta_{\mathrm{C} 1-\alpha \mathrm{KG}}=172.6\right.$ p.p.m. $)$, hyperpolarized $\left[1{ }^{13} \mathrm{C}\right] \alpha-\mathrm{KG}$ hydrate $\left(\delta_{\alpha \mathrm{KG}-\mathrm{H}}=180.9\right.$ p.p.m. $)$ and the resonance of hyperpolarized $\left[1-{ }^{13} \mathrm{C}\right] 2-\mathrm{HG} /\left[5^{-13} \mathrm{C}\right] \alpha-\mathrm{KG}\left(\delta=184\right.$ p.p.m.) are visible. (c) Integral of hyperpolarized $\left[1-{ }^{13} \mathrm{C}\right] \alpha-\mathrm{KG}$ as a function of time after its intravenous injection as measured from slab dynamic ${ }^{13} \mathrm{C}$ spectra acquired in U87IDHmut (black, $n=3$ ) and U87IDHwt (grey, $n=2$ ) tumour-bearing animals (error bars: \pm s.d.). (d) Integral of the 184 -p.p.m. resonance $\left(\left[1-{ }^{13} \mathrm{C}\right] 2-\mathrm{HG} /\left[5-{ }^{13} \mathrm{C}\right] \alpha-\mathrm{KG}\right)$ as measured from slab dynamic ${ }^{13} \mathrm{C}$ spectra acquired in U87IDHmut (black, $n=3$ ) and U87IDHwt (grey, $n=2$ ) tumour-bearing animals (error bars: \pm s.d.). The bimodal temporal evolution of the composite resonance in U87IDHmut suggests metabolic production of hyperpolarized $\left[1-{ }^{13} \mathrm{C}\right] 2-\mathrm{HG}$.

this approach because no models of clinical origin were available when we initiated our study. In fact, to date, only one tumour-forming model of clinical origin has been described $^{44}$. Importantly, however, the levels of 2 -HG detected in our tumour extracts $\left(9.8 \pm 1.6 \mu \mathrm{molg}^{-1}\right.$ of tumour) were comparable to the levels reported in patients $(5-35 \mu \mathrm{mol}$ of $2-\mathrm{HG}$ per gram $)^{2}$ and studies of this model using ${ }^{1} \mathrm{H}$ MRS at high field strengths (7 and 14.1 Tesla) also detected 2-HG in vivo 22,23 . In light of these findings, we believe that the use of a genetically engineered model was justified for our proof-of-concept imaging study.

Following in vitro injection of hyperpolarized $\left[1-{ }^{13} \mathrm{C}\right] \alpha-\mathrm{KG}$ into cell lysates, hyperpolarized $\left[1-{ }^{13} \mathrm{C}\right] 2-\mathrm{HG}$ at 183.9 p.p.m. could be detected in U87IDHmut but not in U87IDHwt lysates. After intravenous injection, hyperpolarized $\left[1-{ }^{13} \mathrm{C}\right] \alpha-\mathrm{KG}$ was also detected in situ in orthotopic tumours, indicating that the probe was delivered to the brain and crossed the BBB in the tumour region. Some reports suggest that $\alpha-K G$ does not easily permeate the cell membrane ${ }^{45}$, whereas other studies show that brain cells are capable of taking up extracellular $\alpha-K^{46}$. Our results demonstrate that, at least in our model, enough hyperpolarized $\left[1-{ }^{13} \mathrm{C}\right] \alpha-\mathrm{KG}$ permeates the tumour cells in vivo to enable the detection of its conversion to hyperpolarized $\left[1-{ }^{13} \mathrm{C}\right] 2-\mathrm{HG}$ within a time frame compatible with a hyperpolarized ${ }^{13} \mathrm{C}$ MRS experiment. Further, our approach can serve to distinguish between mutant IDH1 and wild-type IDH1 tumours in vivo.

Improvements in the delivery of hyperpolarized $\left[1-{ }^{13} \mathrm{C}\right] \alpha-\mathrm{KG}$, as well as in the detection of hyperpolarized $\left[1-{ }^{13} \mathrm{C}\right] 2-\mathrm{HG}$, could be achieved by using alternative $\alpha$-KG-based DNP probes. In particular, ester derivatives of $\alpha-K G$, including octyl- and benzyl-esters have been reported to permeate the cell membrane more effectively ${ }^{45}$. Such derivatives would lead to increased intracellular $\alpha$-KG concentrations and potentially higher levels of 2-HG, and might also enable studies of isocitrate dehydrogenase 2 within the mitochondria ${ }^{46,47}$. Previous studies indicate that the use of ester derivatives creates additional resonances than could hamper spectral differentiation ${ }^{48}$. However, preliminary studies in our lab investigating $\left[1-{ }^{13} \mathrm{C}\right]$ dimethyl- $\alpha$ KG show that the compound resonates far from our spectral region of interest, and thus would not hamper 2-HG detection. Further, the natural abundance $\left[5-{ }^{13} \mathrm{C}\right]$ resonance of the ester was not detectable in our preliminary data, and, if present, would also be expected to resonate away from $\left[1-{ }^{13} \mathrm{C}\right] 2-\mathrm{HG}$.

Our studies were enhanced by the use of innovative VFA and multi-band ${ }^{13} \mathrm{C}$ MR pulse sequences, which use the $\alpha-K G$ polarization efficiently while maximizing the signal of $\left[1-{ }^{13} \mathrm{C}\right]$ $2-\mathrm{HG}$ and minimizing the signal from $\left[5^{-13} \mathrm{C}\right] \alpha-\mathrm{KG}^{34-37}$. Specifically, $\left[5-{ }^{13} \mathrm{C}\right] \alpha-\mathrm{KG}$ originates from the injected hyperpolarized solution and is consequently present at early time 
a

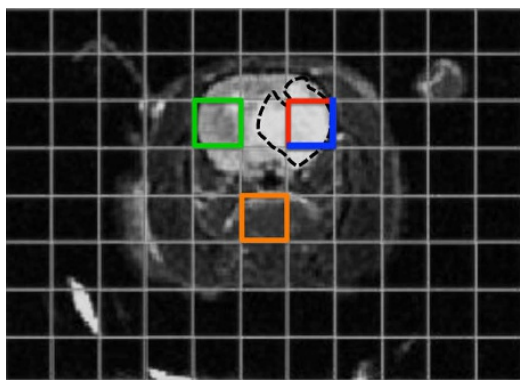

b

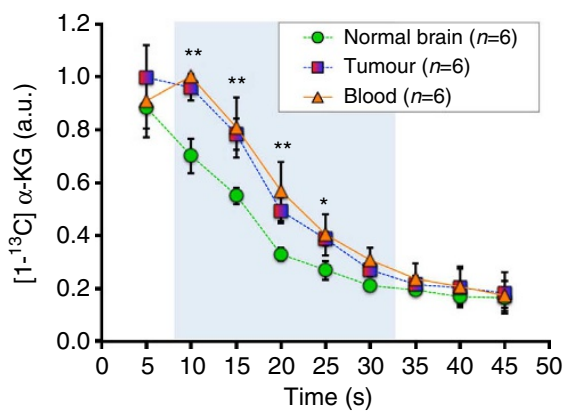

C

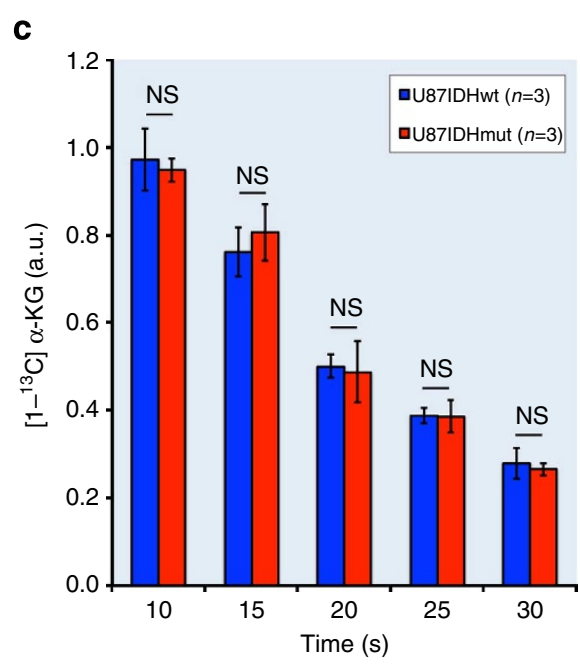

e

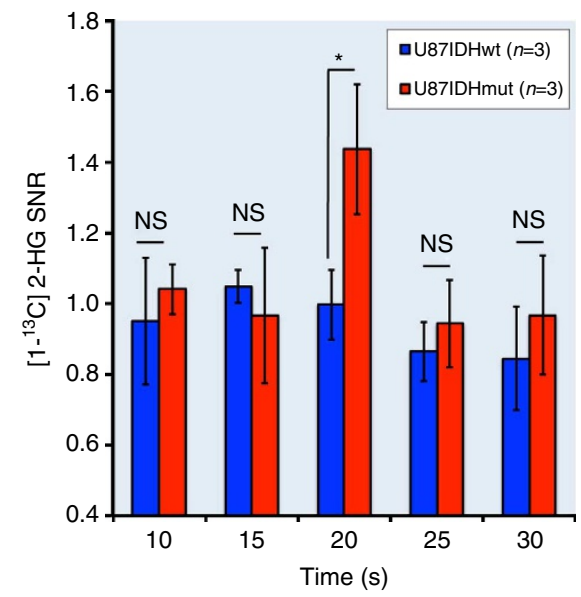

d

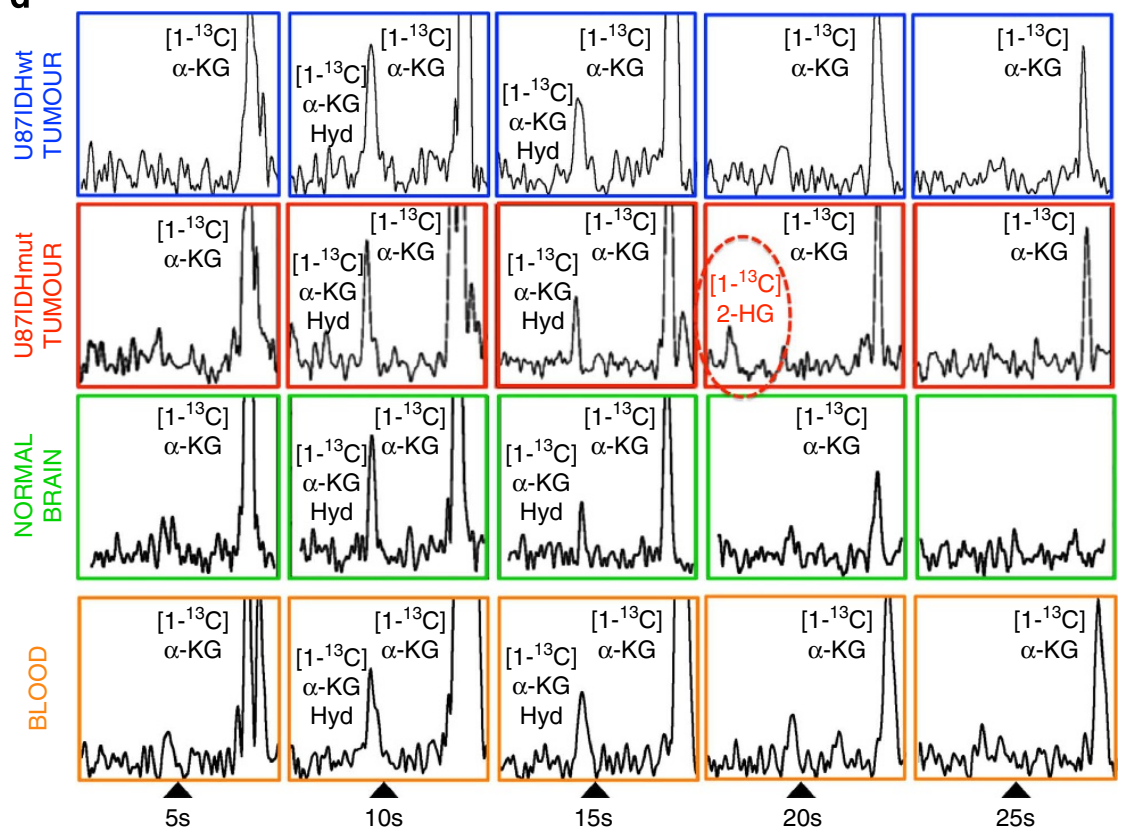

al MR image of U87IDHmut tumour-bearing animal overlaid with the grid used for $2 \mathrm{D}^{13} \mathrm{C} \mathrm{CSI}$ acquisition (red/blue: tumour voxel; orange: blood voxel; green: normal brain voxel). (b) Time courses of hyperpolarized $\left[1-{ }^{13} \mathrm{C}\right] \alpha-K G$ measured in tumour voxels (red/blue: $n=6$, including $n=3$ U87IDHmut and $n=3$ U87IDHwt), in normal brain voxels (green: $n=6$ ) and in blood-containing voxels centred on the basal artery (orange: $n=6$; error bars: \pm s.d.). Significantly higher levels of hyperpolarized [1-13 $C$ ] $\alpha-K G$ were observed in the tumour voxels as compared with normal brain (unpaired Student's $t$-test: ${ }^{\star \star} P<0.005$ at $10,15,20$ and $25 \mathrm{~s} ;{ }^{\star} P<0.05$ at $30 \mathrm{~s}$ ). (c) Levels of hyperpolarized $\left[1-{ }^{13} \mathrm{C}\right] \alpha-K G$ measured in U87IDHwt (blue, $n=3$ ) and U87IDHmut (red, $n=3$ ) tumour voxels at 10, 15, 20, 25 and $30 \mathrm{~s}$ (error bars: \pm s.d.). No significant difference was observed at any time point. (d) Hyperpolarized ${ }^{13} \mathrm{C}$ MR spectra from one U87IDHwt tumour voxel (blue), one U87IDHmut tumour voxel (red), one normal brain voxel (green) and one blood voxel (orange) showing the temporal evolution of metabolites (no averaging performed; all spectra normalized to noise level). A resonance at 184 p.p.m. was observed at $20 \mathrm{~s}$ post injection of hyperpolarized $\left[1{ }^{13} \mathrm{C}\right] \alpha-K G$ only in the IDH1-mutant tumour voxel. (Note: the data presented from the U87IDHmut tumour, blood and normal brain voxels correspond to the U87IDHmut animal presented in a; the data from the U87IDHwt tumour are from an animal for which the MR image is not shown; Hyd, hydrate) (e) SNR of the 184-p.p.m. resonance measured in U87IDHwt (blue) and U87IDHmut (red) tumour voxels at 10, 15, 20, 25 and 30 s (error bars: \pm s.d.). A significant difference was found at 20 s post injection: $S N R=1.4 \pm 0.2$ in U87IDHmut tumours $(n=3)$ versus $S N R=1.1 \pm 0.1$ in U87IDHwt tumours $\left(n=3\right.$, unpaired Student's $t$-test: $\left.{ }^{\star} P<0.05\right)$ consistent with this resonance corresponding to $\left[1-{ }^{13} \mathrm{C}\right] 2-\mathrm{HG}$.

points. In contrast, $\left[1-{ }^{13} \mathrm{C}\right] 2-\mathrm{HG}$ is produced metabolically, and thus builds up at later time points. In our study, the multi-band sequence preserved the magnetization of $\left[1{ }^{13} \mathrm{C}\right] \alpha-\mathrm{KG}$ by using a relatively low FA for its frequency but quenched the polarization of $\left[5^{-13} \mathrm{C}\right] \alpha-\mathrm{KG}$ at the early time points by using a higher FA in that region. Further, the VFA scheme enhanced the signal of $\left[1-{ }^{13} \mathrm{C}\right] 2-\mathrm{HG}$ at the later time points by increasing the FA over time. The use of such sequences is critical for enabling the direct detection of hyperpolarized $\left[1-{ }^{13} \mathrm{C}\right] 2-\mathrm{HG}$ in vivo.

When considering the possibility of future clinical translation, the dose of hyperpolarized $\alpha-K G$ needs to be considered. In the case of hyperpolarized pyruvate, the phase I clinical trial recently completed at $\mathrm{UCSF}^{33}$ was based on a large number of animal studies in which $2 \mathrm{ml}$ of $80 \mathrm{mM}$ hyperpolarized pyruvate $\left(\sim 10 \mathrm{ml} \mathrm{kg}^{-1}, 0.8 \mathrm{mmoles}\right.$ of pyruvate per $\left.\mathrm{kg}\right)$ was injected in rats $^{49}$, and a comparable dose of $0.3 \mathrm{ml}$ of $80 \mathrm{mM}\left(\sim 10 \mathrm{ml} \mathrm{kg}^{-1}\right.$, $0.8 \mathrm{mmoles}$ of pyruvate per $\mathrm{kg}$ ) was used in over 200 studies in mice, all with no adverse effects. In the clinical trial, the rodent studies were scaled in patients to a dose of $0.42 \mathrm{ml} \mathrm{kg}^{-1}$ of $250 \mathrm{mM}$ pyruvate $(0.1 \mathrm{mmoles}$ of pyruvate per $\mathrm{kg})$. Thus, volumes of $30-40 \mathrm{ml}$ were injected, representing $1 \%$ of the total blood volume. These doses were very well tolerated with no doselimiting toxicities and resulted in highly significant imaging results with a spatial resolution as low as $1 \mathrm{~cm}$ isotropic. In the case of $\alpha-K G$, the concentration of hyperpolarized compound in our studies was limited by the technical requirements of the 
a
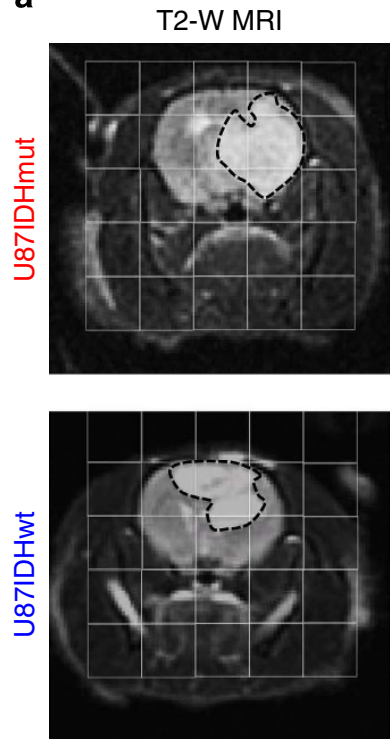

b

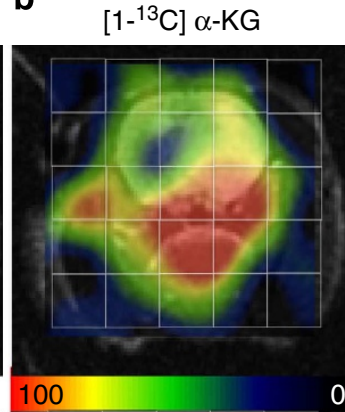

C

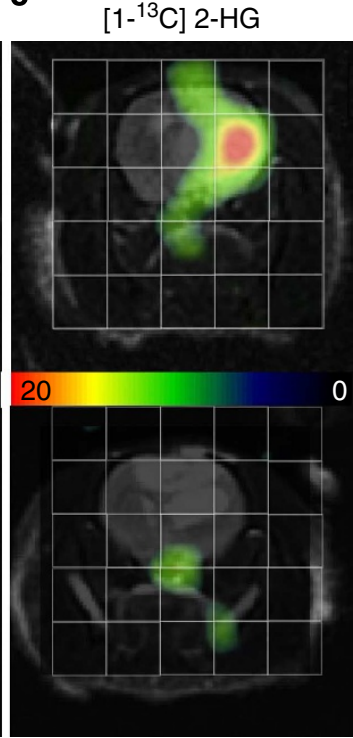

Figure 6 | Hyperpolarized 2-HG is detected only in mutant IDH1 tumours. (a) T2-weighted anatomical MR image of the head of a U87IDHmut (top) and a U87IDHwt (bottom) tumour-bearing animal overlaid with the grid used for $2 \mathrm{D}{ }^{13} \mathrm{C} \mathrm{CSI}$ acquisition. The tumours appear as hyperintense

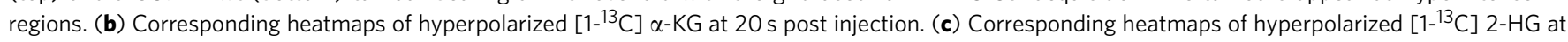
$20 \mathrm{~s}$ post injection, illustrating the presence of this metabolite in IDH1-mutant tumours only.
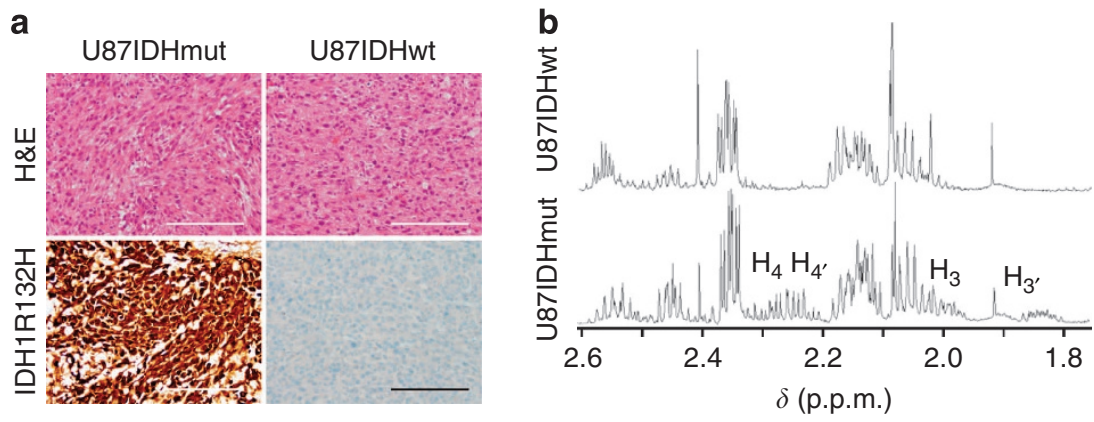

Figure 7 | Post-mortem analysis confirms the presence of mutant IDH1 and 2-HG. (a) U87IDHmut (left) and U87IDHwt (right) tumours had a similar histological appearance and were densely cellular on haematoxylin and eosin staining (H\&E, top row). Immunostaining for mutant IDH1 (IDH1 ${ }^{\mathrm{R} 132 \mathrm{H}}$, bottom row) demonstrated robust, diffuse positivity in U87IDHmut (left) tumours and no positive staining in U87IDHwt (right) tumours (magnification, $\times 200$; scale bar, $100 \mu \mathrm{m}$ ). (b) ${ }^{1} \mathrm{H}$ MR spectrum of U87IDHwt (top) and U87IDHmut (bottom) tumour lysates acquired at 14 Tesla. The multiplets characteristic of $2-H G$ can be seen in the U87IDHmut spectrum not in the U87IDHwt $\left(H_{3^{\prime}}=1.85\right.$ p.p.m.; $H_{3}=2.00$ p.p.m.; $H_{4,4^{\prime}}=2.25$ p.p.m.; see Fig. 2 for proton assignments). The level of $2-\mathrm{HG}$ in U87IDHmut tumour extracts was $9.8 \pm 1.6 \mu \mathrm{mol} \mathrm{g}^{-1}$ of tumour $(n=3)$.

Hypersense hyperpolarizer (maximum volumes of substrate and dissolution buffer) resulting in a maximum achievable concentration of injected $\alpha-\mathrm{KG}$ of $100 \mathrm{mM}$. However, the dose of $\alpha-\mathrm{KG}$ used in this study $\left(0.1 \mathrm{~g} \mathrm{~kg}^{-1}\right.$ body weight $)$ was 6.8 times lower (for a 200-g rat) than the no observed adverse effect level reported in rodents $\left(\mathrm{NOAEL}=1 \mathrm{~g} \mathrm{~kg}^{-1} \text { body weight }\right)^{50}$, and $\alpha-\mathrm{KG}$ is sold in the United States as an over-the-counter nutritional supplement. Consistent with these facts, no adverse effects were observed in any of the animals included in our study $(n=11)$ and higher levels of $\alpha-K G$ could potentially be injected. However, as in the case of pyruvate, the concentration of $\alpha$-KG would need to be increased to perform a similar study in patients with acceptable injection volumes.

Recent studies demonstrate that total 2-HG levels can be monitored in vivo using $1 \mathrm{D}$ or $2 \mathrm{D}^{1} \mathrm{H}$ MRS in patients at clinical field strengths (1.5-3 Tesla) ${ }^{18-21}$. In an effort to compare ${ }^{1} \mathrm{H}$ MRS with our hyperpolarized ${ }^{13} \mathrm{C}$ approach for a potential clinical study, we performed an additional experiment, in which we compared the sensitivity of our rodent coil with the sensitivity of a ${ }^{13} \mathrm{C}$ human head coil array. The sensitivity of the human coil was lower by a factor of $\sim 9$. However, reported ${ }^{1} \mathrm{H}$ MRS studies were performed in voxel sizes of either $8 \mathrm{~cm}^{3}$, that is, $\sim 16$ times larger than our study (no SNR reported in postprocessed data) ${ }^{19,20}$ or $27 \mathrm{~cm}^{3}$, that is, $\sim 54$ times larger than our study $(\mathrm{SNR}=5)^{18}$. With such voxel sizes, we estimate that we would achieve an SNR of $\sim 2.5$ and $\sim 8.4$ on hyperpolarized $\left[1-{ }^{13} \mathrm{C}\right] 2-\mathrm{HG}$.

Nonetheless, before clinical translation can be achieved, the approach presented in this proof-of-concept study will probably require further optimization to enhance $\left[1-{ }^{13} \mathrm{C}\right] 2-\mathrm{HG}$ detection. Several areas of improvement can be envisaged. As mentioned above, the SpinLab clinical polarizer has been shown to enhance the achievable polarization levels ${ }^{42,43}$. This would increase the SNR of our hyperpolarized species, lead to better time-resolved 
kinetics and thus improve the detection of $\left[1{ }^{13} \mathrm{C}\right] 2$-HG. Higher concentrations of $\left[1-{ }^{13} \mathrm{C}\right] \alpha-\mathrm{KG}$, or a more BBB and cellpermeable derivative of $\alpha-\mathrm{KG}$, could result in increased amounts of $\left[1-{ }^{13} \mathrm{C}\right] \alpha$-KG delivered to the tumour and, in turn, greater production of $\left[1-{ }^{13} \mathrm{C}\right]$ 2-HG (until the IDH1 enzyme is saturated). Finally, pulse sequences that would enhance the SNR of hyperpolarized $\left[1-{ }^{13} \mathrm{C}\right] 2-\mathrm{HG}$ can be implemented. A non-dynamic $2 \mathrm{D}$ CSI sequence with a 90 -degree FA at $20 \mathrm{~s}$ post injection would generate a higher SNR by a factor of $\sim 3$ compared with our current study, albeit at the detriment of temporal information. More promising is the recently developed 2D CSI sequence combining the multi-band and VFA scheme ${ }^{35}$, which would enhance the signal of $\left[1^{13} \mathrm{C}\right] 2$-HG by using a progressively increasing FA. In addition, ${ }^{1} \mathrm{H}$ decoupling could be implemented to further increase the in vivo SNR of $\left[1-{ }^{13} \mathrm{C}\right] 2-\mathrm{HG}$, as previously reported ${ }^{51}$.

Importantly, the information obtained by ${ }^{13} \mathrm{C}$ MRS is somewhat different from that obtained by ${ }^{1} \mathrm{H}$ MRS. ${ }^{1} \mathrm{H}$ MRS detects the total, steady-state, 2-HG levels present in both the extracellular and intracellular compartments ${ }^{2}$. In contrast, ${ }^{13} \mathrm{C}$ MRS dynamically informs on the metabolic fate of hyperpolarized $\left[1-{ }^{13} \mathrm{C}\right] \alpha$-KG. Accordingly, although we cannot rule out some residual enzymatic activity in necrotic regions ${ }^{52},{ }^{13} \mathrm{C}$ MRS primarily probes the enzymatic activity of mutant IDH1 in live, metabolically active cells, and, as such, can inform in real-time on the presence of active mutant IDH1, 2-HG synthesis and its potential inhibition by novel therapies ${ }^{14,15}$.

In summary, in this proof-of-concept study, we used ${ }^{13} \mathrm{C}$ MRS and were able to monitor the conversion of hyperpolarized $\left[1-{ }^{13} \mathrm{C}\right] \alpha$-KG into hyperpolarized $\left[1-{ }^{13} \mathrm{C}\right] 2-\mathrm{HG}$ in cell lysates and, more importantly, in vivo in orthotopic brain tumours. Hyperpolarized $\left[1-{ }^{13} \mathrm{C}\right] \alpha$-KG presents a promising, non-radioactive and potentially clinically relevant agent for interrogation of IDH1 status in vivo. This innovative method provides information on IDH1 mutational status and activity in situ, it informs on real-time 2 -HG production and complements the ${ }^{1} \mathrm{H}$ MRS methods that detect total steady-state 2-HG levels ${ }^{18-20}$. Additional optimization is required before clinical translation. Nonetheless, ${ }^{13} \mathrm{C}$ MRS of hyperpolarized $\left[1-{ }^{13} \mathrm{C}\right] \alpha$-KG could ultimately provide an additional valuable tool for diagnosis, prognosis, patient stratification, drug development and studies of response to emerging mutant IDH1-targeted therapies.

\section{Methods \\ Hyperpolarization of $\alpha$-ketoglutarate. $\left[1-{ }^{13} \mathrm{C}\right] \alpha$-ketoglutaric acid (2-oxoglutaric acid, Sigma-Aldrich, St Louis, MO) was dissolved in a mixture of water and gly- cerol (3:1 in volume), with $17.3 \mathrm{mM}$ of OX63 trityl radical and $0.4 \mathrm{mM}$ of Dotarem (Guerbet, France), to a final concentration of $5.9 \mathrm{M}$. A volume of $6.4 \mu \mathrm{l}$ (cell lysate experiments) or $53 \mu \mathrm{l}$ (in vivo experiments) was polarized using a Hypersense polarizer (Oxford Instruments, Oxfordshire, UK) for $\sim 1 \mathrm{~h}(3.35$ Tesla, $1.4 \mathrm{~K}$, $94.089 \mathrm{GHz})$. Following polarization, hyperpolarized $\left[1-{ }^{13} \mathrm{C}\right] \alpha-\mathrm{KG}$ was rapidly dissolved in a Tris-based isotonic buffer $(\mathrm{pH}=7.5 \pm 0.1)$ and used in this form for all experiments.}

Relaxation times and polarization levels. A volume of $20 \mu \mathrm{l}$ of $\left[1-{ }^{13} \mathrm{C}\right] \alpha-\mathrm{KG}$ was polarized as described above. Immediately after dissolution in an isotonic Trisbased buffer ( $40 \mathrm{mM}$ Tris, $80 \mathrm{mM} \mathrm{NaOH}, 0.1 \mathrm{mgl}^{-1} \mathrm{Na}_{2}$ EDTA) to obtain a $40-\mathrm{mM}$ solution buffered at physiological $\mathrm{pH}$, hyperpolarized $\left[1-{ }^{13} \mathrm{C}\right] \alpha-\mathrm{KG}$ was placed in a 5 -ml syringe $(n=3,3$ Tesla Signa clinical MR system, GE Healthcare, Waukesha, WI) or in a $10-\mathrm{mm}$ NMR tube $(n=3,11.7$ Tesla INOVA spectrometer, Agilent Technologies, Inc., Palo Alto, $\mathrm{CA}$ ) and ${ }^{13} \mathrm{C}$ spectra were acquired using a 5-degree $\mathrm{FA}$ and a repetition time $\mathrm{TR}=3 \mathrm{~s}$ (parameters at 3 Tesla: number of transients $(\mathrm{NT})=64$, spectral width $\mathrm{SW}=5 \mathrm{kHz}, 2,048$ points; parameters at 11.7 Tesla: $\mathrm{NT}=300, \mathrm{SW}=20 \mathrm{kHz}, 4,000$ points). For each field strength, the $T_{1}$ relaxation times of hyperpolarized $\left[1{ }^{13} \mathrm{C}\right] \alpha-\mathrm{KG}\left(\delta_{\mathrm{Cl}-\alpha \mathrm{KG}}=172.6\right.$ p.p.m. $)$, hyperpolarized $\left[1-{ }^{13} \mathrm{C}\right] \alpha-\mathrm{KG}$ hydrate $\left(\delta_{\alpha \mathrm{KG}-\mathrm{H}}=180.9\right.$ p.p.m. $)$ and hyperpolarized $\left[5-{ }^{13} \mathrm{C}\right] \alpha-\mathrm{KG}\left(\delta_{\mathrm{C} 5-\alpha \mathrm{KG}}=184\right.$ p.p.m. $)$ were determined by performing a monoexponential fit to the signal decay curve of the hyperpolarized compound ${ }^{53}$.

Following complete decay of the hyperpolarized signal, a thermal spectrum with nearly identical parameters was acquired at 3 Tesla using a 90-degree FA and a repetition time of greater than $4 \times T_{1}\left(\mathrm{TR}=10 \mathrm{~s}, \mathrm{NT}=64 ; T_{1}\right.$ of solution shortened by adding $50 \mu \mathrm{l}$ of Magnevist (Bayer Healthcare, Shawnee Mission, KS) to the hyperpolarized solution). The level of polarization in solution was calculated by comparing the first hyperpolarized spectrum with its corresponding thermal spectrum, correcting for $F A$ and number of scans. The amplitude of the hyperpolarized signal was also corrected for $T_{1}$ relaxation during the transfer time from the polarizer to the spectrometer $(\sim 20 \mathrm{~s})$.

To evaluate the relaxation time $T_{1}$ of $\left[1-{ }^{13} \mathrm{C}\right] 2-\mathrm{HG}$, unlabelled L-2hydroxyglutaric acid (Sigma-Aldrich) was dissolved in a mixture of water and glycerol (6:1 in volume), together with $17.3 \mathrm{mM}$ of OX63 trityl radical and $0.4 \mathrm{mM}$ of Dotarem (Guerbet). A volume of $100 \mu \mathrm{l}$ was polarized using the Hypersense polarizer using the same conditions as for $\left[1{ }^{13} \mathrm{C}\right] \alpha$-KG. After dissolution in $4 \mathrm{ml}$ of Tris-based buffer (final $\mathrm{pH}=7.6$ ),${ }^{13} \mathrm{C}$ MR spectra of hyperpolarized 2-HG were recorded using the same parameters as the ones used for $\alpha$-KG, except that the $F A$ was $10^{\circ}$ instead of $5^{\circ}$. Although the compound was not ${ }^{13} \mathrm{C}$-enriched on the $\mathrm{C} 1$ position, the $1.1 \%$ natural abundance of ${ }^{13} \mathrm{C}$ was sufficient to generate a detectable hyperpolarized resonance at 183.9 p.p.m. The relaxation time $T_{1}$ of $\left[1-{ }^{13} \mathrm{C}\right] 2-\mathrm{HG}$ at 3 and 11.7 Tesla was calculated as described above.

Cell models. Wild-type human IDH1 complementary DNA (MHS1010-58017, Open Biosystems, ThermoScientific) was mutagenized to generate an $\mathrm{R} 132 \mathrm{H}-$ encoding cDNA using site-directed mutagenesis (Quick Change II, Applied Biosystems). Wild-type and mutant IDH1 cDNAs were then sub-cloned into separate green fluorescent protein-encoding lentiviral expression vectors. U87 GBM cells were obtained from the American Tissue Culture Collection and cells expressing exogenous wild-type or mutant IDH1 were created by introduction of lentiviral constructs encoding either the wild-type (U87IDHwt) or R132H IDH1

(U87IDHmut) cDNA, followed by selection of green fluorescent protein-positive cells by flow cytometry and verification for expression of the wild-type or mutan proteins by western blot using wild-type or R132H IDH-specific antibodies. Both modified U87 strains were cultured under standard conditions in high-glucose DMEM, (DMEM H-21, UCSF Cell Culture Facility, San Francisco, CA) supplemented with $10 \%$ heat-inactivated fetal bovine serum (Thermo Scientific Hyclone, Logan, UT), $2 \mathrm{mM}$ L-Glutamine (Invitrogen, Carlsbad, CA), $100 \mathrm{U} \mathrm{ml}^{-1}$ penicillin and $100 \mathrm{mg} \mathrm{ml}^{-1}$ streptomycin (UCSF Cell Culture Facility). All cell lines in culture were maintained as exponentially growing monolayers at $37^{\circ} \mathrm{C}$ in a humidified atmosphere of $95 \%$ air and $5 \% \mathrm{CO}_{2}$.

Western blotting. Western blot analysis was performed on U87IDHmut and U87IDHwt cell lines to monitor IDH1 protein levels. Cytoplasmic proteins were electrophoresed on NuPAGE Novex 4-12\% Bis-Tris gels (Life Technologies, Grand Island, NY) using the SDS-polyacrylamide gel electrophoresis method and electrotransferred onto polyvinylidene difluoride membranes. Blots were blocked and incubated with primary antibodies anti-IDH1wt (rabbit, number 3997, 1:1,000 dilution overnight; Cell Signaling Technology, Danvers MA) and anti-IDH1 ${ }^{\mathrm{R} 132 \mathrm{H}}$ (mouse monoclonal H09 clone, 1:1,000 dilution overnight; Dianova, Miami, FL), and were then incubated with secondary antibody either anti-rabbit IgG (DyLight 800, 1:2,000; Cell Signaling Technology) or anti-mouse IgG (DyLight 680, 1:2,000; Cell Signaling Technology). The blots were visualized with a LI-COR Odyssey Infrared Imaging System (LI-COR Biosciences, Lincoln, NE).

Spectrophotometric assay. Mutant IDH1 activity was monitored in U87IDHmut and U87IDHwt cell lysates by adapting a spectrophotometric assay. For each cell type, $\sim 2.0 \times 10^{7}$ cells were lysed in $400 \mu \mathrm{l}$ of $50 \mathrm{mM}$ Tris-based buffer with $1 \mu \mathrm{l} \mathrm{ml}{ }^{-1}$ protease inhibitor cocktail (Sigma-Aldrich). Lysates were then homogenized by passing through a 27 gauge needle and sonicated on ice at $10 \%$ amplitude $(3 \times 5 \mathrm{~s}$ with $10 \mathrm{~s}$ intervals) using an FB505 $20 \mathrm{kHz}$ ultrasonicator (Fisher, Pittsburgh, PA). Cell lysates were centrifuged at 14,000 r.p.m. for $30 \mathrm{~min}$ at $4^{\circ} \mathrm{C}$. The supernatant was added to $1 \mathrm{ml}$ of reaction buffer composed of $33 \mathrm{mM}$ Tris-based buffer, $0.33 \mathrm{mM}$ EDTA, $0.1 \mathrm{mM}$ NADPH, $1.33 \mathrm{mM} \mathrm{MnCl}_{2}$ and $1.3 \mathrm{mM}$ $\alpha$-KG (Sigma-Aldrich). A volume of $100 \mu \mathrm{l}$ per well was then placed in a 96-well plate (Greiner Bio One, Monroe, NC). Absorbance at $\lambda=340 \mathrm{~nm}$ was monitored every $10 \mathrm{~s}$ for $30 \mathrm{~min}$ using an Infinite m200 spectrophotometer (Tecan Systems, Inc., CA) and correlated to the concentration of $\mathrm{NADPH}^{54,55}$ using a previously established calibration curve. For each lysate, the reaction rate $R_{\mathrm{IDHmut}}$ of mutant IDH was calculated by linear regression of the first $300 \mathrm{~s}$ and reported in fmol of NADPH consumed per minute per cell $(n=3$ per cell type).

${ }^{1}$ H MRS of in cell lysates. Approximately $1.5 \times 10^{8}$ U87IDHmut and U87IDHwt cells were extracted using the dual-phase extraction method $^{29} .1 \mathrm{D}{ }^{1} \mathrm{H}$ MRS spectra of U87IDHmut $(n=3)$ and U87IDHwt $(n=3)$ extracts were acquired on a 14Tesla spectrometer (Bruker BioSpin Corporation, Billerica, MA) equipped with a 5-mm broadband probe. For $1 \mathrm{D}{ }^{1} \mathrm{H}$ MRS, the following acquisition parameters were used: 90-degree FA, repetition time $\mathrm{TR}=2 \mathrm{~s}, \mathrm{SW}=7194 \mathrm{~Hz}, 20,000$ points, $\mathrm{NT}=100$. All spectral assignments were based on literature reports (http:// www.hmdb.ca) and confirmed by $2 \mathrm{D}{ }^{1} \mathrm{H}$ MRS (2D total correlation spectroscopy sequence; $\mathrm{f2}$ : $\mathrm{SW}=20,000 \mathrm{~Hz}, 8,192$ points; $\mathrm{f} 1: \mathrm{SW}=6,500 \mathrm{~Hz}, 64$ points; mixing time $40 \mathrm{~ms}$, WURST-8 adiabatic spin lock). 2-HG levels were quantified by peak deconvolution and integration using ACD/SpecManager 9 (Advanced Chemistry 
Development, Toronto, Ontario, Canada), correction for saturation and normalization to a trimethylsilyl propionate (TSP) reference and to cell number (Cambridge Isotope Laboratories, Andover, MA).

Hyperpolarized ${ }^{13} \mathbf{C}$ MR studies of cell lysates. A total of $1.2 \times 10^{8}$ U87IDHmut $(n=3)$ and U87IDHwt $(n=3)$ cells were extracted as described above. Lysates were placed in a $10-\mathrm{mm}$ NMR tube and combined with a reaction buffer of similar composition to that used in the IDH1 spectrophotometric enzyme assay (while maintaining the same substrate concentrations per cell). In addition, $\mathrm{MgCl}_{2}$ was used instead of $\mathrm{MnCl}_{2}$ to prevent $\mathrm{Mn}^{2+}$-induced paramagnetic relaxation of nuclear hyperpolarization. Within $10 \mathrm{~s}$ of combining lysates and buffer, $1.2 \mathrm{ml}$ of hyperpolarized $\left[1-{ }^{13} \mathrm{C}\right] \alpha$-KG dissolved in buffer $(40 \mathrm{mM}$ Tris, $9.4 \mathrm{mM} \mathrm{NaOH}$, $0.1 \mathrm{mgl}^{-1} \mathrm{Na}_{2}$ EDTA) was added to a final concentration of $4.7 \mathrm{mM}$. Immediately after injection, dynamic sets of hyperpolarized ${ }^{13} \mathrm{C}$ spectra were acquired on a 11.7Tesla INOVA spectrometer (Agilent Technologies, Inc.) with a 13-degree FA for $300 \mathrm{~s}(\mathrm{TR}=3 \mathrm{~s}, \mathrm{NT}=100, \mathrm{SW}=20 \mathrm{kHz}, 40,000$ points). After the total decay of the hyperpolarized signal, a $2 \mathrm{D}{ }^{1} \mathrm{H}-{ }^{13} \mathrm{C}$ gradient-selected heteronuclear multiple bond coherence spectrum of U87IDHmut cell lysate was recorded on a 14-Tesla Varian INOVA spectrometer (Agilent Technologies, Inc.) equipped with a $5-\mathrm{mm}$ cryogenically cooled triple resonance inverse probe to confirm the $\left[1-{ }^{13} \mathrm{C}\right] 2-\mathrm{HG}$ assignment: $\mathrm{f} 2 \mathrm{SW}=6903 \mathrm{~Hz}, 2,048$ points; $\mathrm{f} 1$ : $\mathrm{SW}=36,207 \mathrm{~Hz}, 4,000$ points. ${ }^{13} \mathrm{C}$ pulse delays were $3.75 \mathrm{~ms}$ (to filter out one-bond coupling of $140 \mathrm{~Hz}$ ) and $100 \mathrm{~ms}$ (optimal for $5 \mathrm{~Hz}{ }^{1} \mathrm{H}-{ }^{13} \mathrm{C}$ interactions).

Tumour-bearing animals. All animal research was approved by the Institutional Animal Care and Use Committee of the University of California, San Francisco. Athymic rats ( $n=6$ U87IDHmut, $n=5$ U87IDHwt, average weight $200 \mathrm{~g}$, male, $\mathrm{rnu} / \mathrm{rnu}$ homozygous, 5-6-week old at the time of intracranial injection; Harlan Laboratories, Indianapolis, IN) were used in this study. An hour before starting the intracranial injection, U87IDHwt or U87IDHmut cells were washed once with PBS solution, collected by trypsinization, counted and resuspended in serum-free McCoy's medium to a final concentration of $3 \times 10^{5}$ cells per $10 \mu \mathrm{l}$. For intracranial injection, rats were anaesthetized by an intraperitoneal injection of a mixture of ketamine/xylazine $\left(100 / 20 \mathrm{mg} \mathrm{kg}^{-1}\right.$, respectively). A volume of $10 \mu \mathrm{l}$ of cell suspension was slowly injected into the right putamen of the animal brain by freehand technique ${ }^{56}$. Buprenorphine $\left(\right.$ dose $\left.=0.03 \mathrm{mg} \mathrm{kg}^{-1}, V=600 \mu \mathrm{l}\right)$ and bupivacaine (dose $=5 \mathrm{mg} \mathrm{kg}^{-1}, V=300 \mu \mathrm{l}$ ) were injected subcutaneously right before injection of tumour cells for optimal pain management.

In vivo-hyperpolarized ${ }^{\mathbf{1 3}} \mathbf{C}$ MR studies. In vivo experiments were performed on a 3-Tesla clinical MR system (GE Healthcare) equipped with a dual-tuned ${ }^{1} \mathrm{H}-{ }^{13} \mathrm{C}$ transmit/receive volume coil $\left(\emptyset_{\mathrm{I}}=40 \mathrm{~mm}\right)$. Rats were anaesthetized using isoflurane $\left(1-2 \%\right.$ in $\left.\mathrm{O}_{2}, 1.5 \mathrm{lmin}^{-1}\right)$ and a 23 gauge catheter was secured in the tail vein for injection of hyperpolarized material.

Anatomical imaging was performed to assess tumour location (2D fast spin echo, axial/coronal, TE/TR $=20 / 1,200 \mathrm{~ms}$, field of view $=40 \times 40 \mathrm{~mm}^{2}$, matrix $256 \times 256,20$ slices, thickness $=1 \mathrm{~mm}$, acquisition time $5 \mathrm{~min} 7 \mathrm{~s}, \mathrm{NT}=1)$. After $1 \mathrm{~h}$, hyperpolarized $\left[1-{ }^{13} \mathrm{C}\right] \alpha-\mathrm{KG}$ was rapidly dissolved in isotonic buffer $(40 \mathrm{mM}$ Tris, $200 \mathrm{mM} \mathrm{NaOH}, 0.1 \mathrm{mgl}^{-1} \mathrm{Na}_{2}$ EDTA) to obtain a $100-\mathrm{mM}$ solution. Intravenous injection began within $<20 \mathrm{~s}$ after dissolution. A volume of $2 \mathrm{ml}$ of the hyperpolarized solution was injected over $12 \mathrm{~s}$.

Dynamic ${ }^{13} \mathrm{C}$ MR spectra were acquired from a 2-cm slab including the whole tumour on U87IDHwt $(n=2)$ and U87IDHmut $(n=3)$ tumour-bearing animals. The custom radiofrequency (RF) pulses were limited to this thickness by the demanding spectral and spatial selection requirements combined with gradient and RF hardware limitations of the clinical scanner. Nonetheless, this slice thickness provided full coverage for all tumours, which usually had an ellipsoid shape and grew preferentially along the anterior-posterior direction. The slab was positioned to minimize the contributions of neck and nose blood vessels. Data were acquired using an adiabatic double spin-echo sequence (TE/TR $=35 / 3,000 \mathrm{~ms})^{36}$. It used multi-band spectral-spatial RF excitation pulses with a VFA scheme that started with a smaller FA for $\left[1-{ }^{13} \mathrm{C}\right] \alpha-\mathrm{KG}\left(3.5^{\circ}, \mathrm{VFA} 1\right)$ to preserve substrate magnetization and a larger FA for $\left[1-{ }^{13} \mathrm{C}\right] 2-\mathrm{HG} /\left[5^{-13} \mathrm{C}\right] \alpha-\mathrm{KG} /\left[1{ }^{13} \mathrm{C}\right] \alpha-\mathrm{KG}$ hydrate $\left(42.3^{\circ}, \mathrm{VFA} 2\right)$ for improved SNR of $2-\mathrm{HG}^{34}$. These FAs were progressively increased over time to efficiently use the hyperpolarized magnetization in the presence of metabolic conversion, with both VFA1 and VFA2 ramping up to a final 90 -degree $\mathrm{FA}^{35}$. FA and frequency calibration was performed on a $0.6-\mathrm{ml}$ syringe of $8 \mathrm{M}\left[1-{ }^{13} \mathrm{C}\right]$ urea placed next to the head.

${ }^{13} \mathrm{C} 2 \mathrm{D}$ dynamic CSI was acquired starting $5 \mathrm{~s}$ after the beginning of hyperpolarized $\left[1-{ }^{13} \mathrm{C}\right] \alpha-\mathrm{KG}$ injection in U87IDHwt $(n=3)$ and U87IDHmut $(n=3)$ animals using a sequence optimized for $\alpha-\mathrm{KG} / 2-\mathrm{HG}^{34,37}$. This optimization included using a specialized multi-band spectral-spatial RF excitation pulse designed to provide a 4 -degree FA for $\left[1-{ }^{13} \mathrm{C}\right] \alpha-\mathrm{KG}$ (to preserve substrate magnetization) and $25^{\circ}$ elsewhere for improved SNR for 2-HG. An echo-planar spectroscopic imaging read-out gradient was used for increased imaging speed. This improves the SNR by reducing artifacts from $T_{1}$ decay, metabolic conversion and motion that can cause signal loss and blurring. The echo-planar spectroscopic imaging was optimized for maximum SNR efficiency while meeting the spectral bandwidth and spatial resolution requirements. Additional sequence parameters were adiabatic double spin-echo acquisition, bandwidth $543 \mathrm{~Hz}$, resolution $10.4 \mathrm{~Hz}$, 52 points, $\mathrm{TE} / \mathrm{TR}=140 / 215 \mathrm{~ms}$, matrix $8 \times 18,5 \times 5 \mathrm{~mm}^{2}$ resolution, slice thickness $2 \mathrm{~cm}, 1.7 \mathrm{~s}$ per image, images every $5 \mathrm{~s}$.

For all in vivo studies, $\mathrm{B}_{0}$ shimming was performed using the automatic GE shimming routine, which acquires three orthogonal field maps and uses a least squares fit to set the linear shim gradients. The linewidth of $\left[1-{ }^{13} \mathrm{C}\right] \alpha-\mathrm{KG}$ was $26.3 \pm 9.8 \mathrm{~Hz}$ for the 1D MRS experiment (entire slab) and $23.0 \pm 3.4 \mathrm{~Hz}$ for the $2 \mathrm{D}$ CSI (brain voxel). ${ }^{1} \mathrm{H}$ decoupling was not applied; however, its use could increase the in vivo SNR of $\left[1-{ }^{13} \mathrm{C}\right] 2-\mathrm{HG}$ in future studies, as illustrated by Marjanska et al. ${ }^{51}$

In vivo MR data analysis. Tumour volume was assessed from the anatomical T2 weighted images using the dedicated in-house SIVIC software (http://www.sourceforge.net/projects/sivic/). For each animal, manual contouring of the tumour was performed for all axial slices and linear extrapolation was used to estimate final tumour volume.

For in vivo slab dynamic experiments, integrals of hyperpolarized $\left[1-{ }^{13} \mathrm{C}\right] \alpha-\mathrm{KG}$ and $\left[1-{ }^{13} \mathrm{C}\right] 2-\mathrm{HG}$ were quantified by peak integration of the dynamic hyperpolarized ${ }^{13} \mathrm{C}$ spectra using $\mathrm{ACD} /$ SpecManager 9 (Advanced Chemistry Development). The integrals were then corrected for the VFA schemes using Matlab (Mathworks, Natick, MA).

For in vivo $2 \mathrm{D}$ CSI experiments, ${ }^{13} \mathrm{C}$ data sets were processed using SIVIC software to calculate the integrals of hyperpolarized $\left[1-{ }^{13} \mathrm{C}\right] \alpha-\mathrm{KG}$ and $\left[1-{ }^{13} \mathrm{C}\right] 2$ HG and the s.d. of the noise to derive each metabolite SNR value. Superimposing the $2 \mathrm{D}{ }^{13} \mathrm{C}$ CSI data onto the axial T2-weighted images was performed to localize the tumour voxels (estimated $68 \pm 27 \%$ of tumour tissue). Colour heatmaps of hyperpolarized $\left[1-{ }^{13} \mathrm{C}\right] \alpha-\mathrm{KG}$ and $\left[1-{ }^{13} \mathrm{C}\right] 2-\mathrm{HG}$ were generated at $20 \mathrm{~s}$ after the start of injection by sinc-based spatial interpolation of the $2 \mathrm{D}{ }^{13} \mathrm{C}$ CSI data to the anatomical image resolution using SIVIC.

Post-mortem analysis of tumours. One hour after the end of the last hyperpolarized ${ }^{13} \mathrm{C}$ MR experiment, animals ( $n=5$ U87IDHmut, $n=4$ U87IDHwt) were euthanized. Brains were resected and sectioned in the coronal plane to bisect the tumour.

The posterior sections of the brains were placed in $10 \%$ buffered formalin for $24 \mathrm{~h}$, and then moved to $70 \%$ cold ethanol. The sections were then embedded in paraffin and sectioned in the axial plane. Sections were stained with haematoxylin and eosin and immunostained for mutant IDH1 (IDH1 ${ }^{\mathrm{R} 132 \mathrm{H}}$ ) using a mouse monoclonal anti-IDH1 $1^{\mathrm{R} 132 \mathrm{H}}$ (DIA H09; Dianova) at 1:50 dilution. All stained slides were imaged using an Olympus BX41 microscope and Olympus DP72 camera and assessed qualitatively.

The anterior sections of the brains were dissected to isolate the tumour from the normal brain tissue, and tumours were quickly snap-frozen in liquid $\mathrm{N}_{2}$. Tumour tissues were then extracted using the dual-phase extraction method ${ }^{57}$. After lyophilization, tumour extracts were reconstituted in $400 \mu \mathrm{D}_{2} \mathrm{O}$ in a 5-mm NMR tube and $1 \mathrm{D}{ }^{1} \mathrm{H}$ MR spectra were recorded on a on a 14-Tesla Varian INOVA spectrometer (Agilent Technologies, Inc.) using the following parameters: 90degree $\mathrm{FA}, \mathrm{TR}=2 \mathrm{~s}, \mathrm{SW}=7194 \mathrm{~Hz}, 20,000$ points, $\mathrm{NT}=128$. In vivo 2 -HG levels were quantified by peak deconvolution and integration using ACD/SpecManager 9 (Advanced Chemistry Development), correction for saturation and normalization to a TSP reference and to grams of tumour tissue (Cambridge Isotope Laboratories).

Coil sensitivity in rodents versus humans. In an effort to estimate the achievable SNR of hyperpolarized $\left[1-{ }^{13} \mathrm{C}\right] 2-\mathrm{HG}$ in a patient brain tumour, we needed to account for the decreased sensitivity of the larger coils required for patient studies. To this end, we compared the sensitivity of the rodent coil used in our study with the sensitivity of a human ${ }^{13} \mathrm{C}$ head coil array used for clinical brain imaging (eight-channel custom coil array with two $18 \mathrm{~cm}$ linear panels; four surface coils per panel; rectangular coil element $5 \times 10 \mathrm{~cm})^{58}$. A $0.6-\mathrm{ml}$ sealed syringe containing $8 \mathrm{M}\left[1-{ }^{13} \mathrm{C}\right]$ urea was positioned in the center of each of the two coils and an identical pulse-acquire sequence was applied to measure the $\left[1-{ }^{13} \mathrm{C}\right]$ urea signal (90-degree FA, NT=64). The decrease in sensitivity in the human coil was assessed by direct comparison of the amplitudes of the $\left[1-{ }^{13} \mathrm{C}\right]$ urea signal in human versus rodent coil.

Statistical analysis. All results are expressed as mean \pm s.d. The unpaired twotailed Student's $t$-test was used to determine statistical significance of the results, with a $P$-value $<0.05$ considered as significant.

\section{References}

1. Ward, P. S. et al. The common feature of leukemia-associated IDH1 and IDH2 mutations is a neomorphic enzyme activity converting alpha-ketoglutarate to 2hydroxyglutarate. Cancer Cell 17, 225-234 (2010).

2. Dang, L. et al. Cancer-associated IDH1 mutations produce 2-hydroxyglutarate. Nature 462, 739-744 (2009).

3. Mardis, E. R. et al. Recurring mutations found by sequencing an acute myeloid leukemia genome. N. Engl. J. Med. 361, 1058-1066 (2009). 
4. Ohgaki, H. \& Kleihues, P. The definition of primary and secondary glioblastoma. Clin. Cancer Res. 19, 764-772 (2013).

5. Yang, H., Ye, D., Guan, K. L. \& Xiong, Y. IDH1 and IDH2 mutations in tumorigenesis: mechanistic insights and clinical perspectives. Clin. Cancer Res. 18, 5562-5571 (2012).

6. Losman, J. A. \& Kaelin, Jr. W. G. What a difference a hydroxyl makes: mutant IDH, (R)-2-hydroxyglutarate, and cancer. Genes Dev. 27, 836-852 (2013).

7. Walker, E. J. et al. Monoallelic expression determines oncogenic progression and outcome in benign and malignant brain tumors. Cancer Res. 72, 636-644 (2012).

8. Leonardi, R., Subramanian, C., Jackowski, S. \& Rock, C. O. Cancer-associated isocitrate dehydrogenase mutations inactivate $\mathrm{NADPH}$-dependent reductive carboxylation. J. Biol. Chem. 287, 14615-14620 (2012).

9. Ward, P. S. et al. The potential for isocitrate dehydrogenase mutations to produce 2-hydroxyglutarate depends on allele specificity and subcellular compartmentalization. J. Biol. Chem. 288, 3804-3815 (2013).

10. Turcan, S. et al. IDH1 mutation is sufficient to establish the glioma hypermethylator phenotype. Nature 483, 479-483 (2012).

11. $\mathrm{Xu}, \mathrm{W}$. et al. Oncometabolite 2-hydroxyglutarate is a competitive inhibitor of alpha-ketoglutarate-dependent dioxygenases. Cancer Cell 19, 17-30 (2011).

12. Huse, J. T., Phillips, H. S. \& Brennan, C. W. Molecular subclassification of diffuse gliomas: seeing order in the chaos. Glia 59, 1190-1199 (2011).

13. Lu, C. et al. IDH mutation impairs histone demethylation and results in a block to cell differentiation. Nature 483, 474-478 (2012).

14. Popovici-Muller, J. et al. Discovery of the first potent inhibitors of mutant IDH1 that lower tumor 2-HG in vivo. Acs Med. Chem. Lett. 3, 850-855 (2012).

15. Rohle, D. et al. An inhibitor of mutant IDH1 delays growth and promotes differentiation of glioma cells. Science 340, 626-630 (2013).

16. Nelson, S. J. Assessment of therapeutic response and treatment planning for brain tumors using metabolic and physiological MRI. NMR Biomed. 24, 734-749 (2011).

17. Julia-Sape, M. et al. Prospective diagnostic performance evaluation of singlevoxel $1 \mathrm{H}$ MRS for typing and grading of brain tumours. NMR Biomed. 25, 661-673 (2012).

18. Andronesi, O. C. et al. Detection of 2-hydroxyglutarate in IDH-mutated glioma patients by in vivo spectral-editing and $2 \mathrm{D}$ correlation magnetic resonance spectroscopy. Sci. Transl. Med. 4, $116 \mathrm{ra114}$ (2012).

19. Choi, C. et al. 2-hydroxyglutarate detection by magnetic resonance spectroscopy in IDH-mutated patients with gliomas. Nat. Med. 18, 624-629 (2012).

20. Pope, W. B. et al. Non-invasive detection of 2-hydroxyglutarate and other metabolites in IDH1 mutant glioma patients using magnetic resonance spectroscopy. J. Neurooncol. 107, 197-205 (2012).

21. Esmaeili, M., Vettukattil, R. \& Bathen, T. F. 2-hydroxyglutarate as a magnetic resonance biomarker for glioma subtyping. Transl. Oncol. 6, 92-98 (2013).

22. Lazovic, J. et al. Detection of 2-hydroxyglutaric acid in vivo by proton magnetic resonance spectroscopy in U87 glioma cells overexpressing isocitrate dehydrogenase-1 mutation. Neuro. Oncol. 14, 1465-1472 (2012).

23. Chaumeil, M. M. et al. In vivo comparison of total and hyperpolarized lactate levels assessed by localized 1H MRS and hyperpolarized 13C MRSI in glioblastoma models at 14.1Tesla. in International Society of Magnetic Resonance in Medicine Vol. 4375 Melbourne, 2012).

24. Kalinina, J. et al. Detection of 'oncometabolite' 2-hydroxyglutarate by magnetic resonance analysis as a biomarker of IDH1/2 mutations in glioma. J. Mol. Med. 90, 1161-1171 (2012).

25. Elkhaled, A. et al. Magnetic resonance of 2-hydroxyglutarate in IDH1-mutated low-grade gliomas. Sci. Transl. Med. 4, 116 ra115 (2012).

26. Ardenkjaer-Larsen, J. H. et al. Increase in signal-to-noise ratio of $>10,000$ times in liquid-state NMR. Proc. Natl Acad. Sci. USA 100, 10158-10163 (2003).

27. Kurhanewicz, J. et al. Analysis of cancer metabolism by imaging hyperpolarized nuclei: prospects for translation to clinical research. Neoplasia 13, 81-97 (2011).

28. Day, S. E. et al. Detecting tumor response to treatment using hyperpolarized 13C magnetic resonance imaging and spectroscopy. Nat. Med. 13, 1382-1387 (2007).

29. Ward, C. S. et al. Noninvasive detection of target modulation following phosphatidylinositol 3-kinase inhibition using hyperpolarized 13C magnetic resonance spectroscopy. Cancer Res. 70, 1296-1305 (2010).

30. Park, I. et al. Detection of early response to temozolomide treatment in brain tumors using hyperpolarized 13C MR metabolic imaging. J. Magn. Reson. Imaging 33, 1284-1290 (2011).

31. Chaumeil, M. M. et al. Hyperpolarized 13C MR spectroscopic imaging can be used to monitor Everolimus treatment in vivo in an orthotopic rodent model of glioblastoma. Neuroimage 59, 193-201 (2012).
32. Day, S. E. et al. Detecting response of rat C6 glioma tumors to radiotherapy using hyperpolarized [1- 13C]pyruvate and 13C magnetic resonance spectroscopic imaging. Magn. Reson. Med. 65, 557-563 (2011).

33. Nelson, S. J. et al. Metabolic imaging of patients with prostate cancer using hyperpolarized [1-13C]pyruvate. Sci. Transl. Med. 5, 198 ra108 (2013).

34. Larson, P. E. et al. Multiband excitation pulses for hyperpolarized ${ }^{13} \mathrm{C}$ dynamic chemical-shift imaging. J. Magn. Reson. 194, 121-127 (2008).

35. Xing, Y., Reed, G. D., Pauly, J. M., Kerr, A. B. \& Larson, P. E. Z. Optimal variable flip angle schemes for multi-band dynamic acquisition of hyperpolarized 13C MRSI. J. Magn. Reson. 234, 75-81 (2013).

36. Cunningham, C. H. et al. Double spin-echo sequence for rapid spectroscopic imaging of hyperpolarized ${ }^{13}$ C. J. Magn. Reson. 187, 357-362 (2007).

37. Larson, P. E. et al. Investigation of tumor hyperpolarized $\left[1-{ }^{13} \mathrm{C}\right]$-pyruvate dynamics using time-resolved multiband RF excitation echo-planar MRSI. Magn. Reson. Med. 63, 582-591 (2010).

38. Kazan, S. M. et al. Kinetic modeling of hyperpolarized (13) C pyruvate metabolism in tumors using a measured arterial input function. Magn. Reson. Med. (e-pub ahead of print 20 November 2012; doi:10.1002/mrm.24546).

39. Chen, A. P. et al. Hyperpolarized C-13 spectroscopic imaging of the TRAMP mouse at 3T-initial experience. Magn. Reson. Med. 58, 1099-1106 (2007).

40. Chen, A. P. et al. Feasibility of using hyperpolarized $\left[1-{ }^{13} \mathrm{C}\right]$ lactate as a substrate for in vivo metabolic ${ }^{13} \mathrm{C}$ MRSI studies. Magn. Reson. Imaging 26, 721-726 (2008).

41. $\mathrm{Hu}, \mathrm{S}$. et al. In vivo measurement of normal rat intracellular pyruvate and lactate levels after injection of hyperpolarized [1-(13)C]alanine. Magn. Reson. Imaging 29, 1035-1040 (2011).

42. Ardenkjaer-Larsen, J. H. et al. Dynamic nuclear polarization polarizer for sterile use intent. NMR Biomed. 24, 927-932 (2011).

43. Batel, M. et al. A multi-sample $94 \mathrm{GHz}$ dissolution dynamic-nuclearpolarization system. J. Magn. Reson. 214, 166-174 (2012).

44. Luchman, H. A. et al. An in vivo patient-derived model of endogenous IDH1mutant glioma. Neuro. Oncol. 14, 184-191 (2012).

45. MacKenzie, E. D. et al. Cell-permeating alpha-ketoglutarate derivatives alleviate pseudohypoxia in succinate dehydrogenase-deficient cells. Mol. Cell Biol. 27, 3282-3289 (2007).

46. Shank, R. P. \& Bennett, D. J. 2-Oxoglutarate transport: a potential mechanism for regulating glutamate and tricarboxylic acid cycle intermediates in neurons. Neurochem. Res. 18, 401-410 (1993).

47. Smolkova, K. \& Jezek, P. The role of mitochondrial NADPH-dependent isocitrate dehydrogenase in cancer cells. Int. J. Cell Biol. 2012, 273947 (2012).

48. Hurd, R. E. et al. Metabolic imaging in the anesthetized rat brain using hyperpolarized $\left[1-{ }^{13} \mathrm{C}\right]$ pyruvate and $\left[1-{ }^{13} \mathrm{C}\right]$ ethyl pyruvate. Magn. Reson. Med. 63, 1137-1143 (2010).

49. Park, I. et al. Hyperpolarized ${ }^{13} \mathrm{C}$ magnetic resonance metabolic imaging: application to brain tumors. Neuro. Oncol. 12, 133-144 (2010).

50. Bhattacharya, R., Gujar, N., Singh, P., Rao, P. \& Vijayaraghavan, R. Toxicity of alpha-ketoglutarate following 14-days repeated oral administration in Wistar rats. Cell. Mol. Biol. 57 Suppl OL1543-OL1549 (2011).

51. Marjanska, M. et al. In vivo ${ }^{13} \mathrm{C}$ spectroscopy in the rat brain using hyperpolarized [1-(13)C]pyruvate and [2-(13)C]pyruvate. J. Magn. Reson. 206, 210-218 (2010).

52. Gallagher, F. A. et al. Production of hyperpolarized [1,4-13C2]malate from [1,4-13C2]fumarate is a marker of cell necrosis and treatment response in tumors. Proc. Natl Acad. Sci. USA 106, 19801-19806 (2009).

53. Golman, K., Zandt, R. I., Lerche, M., Pehrson, R. \& Ardenkjaer-Larsen, J. H. Metabolic imaging by hyperpolarized ${ }^{13} \mathrm{C}$ magnetic resonance imaging for in vivo tumor diagnosis. Cancer Res. 66, 10855-10860 (2006).

54. Fatania, H. R., al-Nassar, K. E. \& Thomas, N. Chemical modification of rat liver cytosolic $\mathrm{NADP}(+)$-linked isocitrate dehydrogenase by $\mathrm{N}$-ethylmaleimide. Evidence for essential sulphydryl groups. FEBS Lett. 322, 245-248 (1993).

55. Yan, H. et al. IDH1 and IDH2 mutations in gliomas. N. Engl. J. Med. 360, 765-773 (2009).

56. Ozawa, T. et al. Growth of human glioblastomas as xenografts in the brains of athymic rats. In Vivo 16, 55-60 (2002).

57. Brandes, A. H., Ward, C. S. \& Ronen, S. M. 17-allyamino-17demethoxygeldanamycin treatment results in a magnetic resonance spectroscopy-detectable elevation in choline-containing metabolites associated with increased expression of choline transporter SLC44A1 and phospholipase A2. Breast Cancer Res. 12, R84 (2010).

58. Ohliger, M. A. et al. Combined parallel and partial fourier MR reconstruction for accelerated 8-channel hyperpolarized carbon-13 in vivo magnetic resonance spectroscopic imaging (MRSI). J. Magn. Reson. Imaging. (e-pub ahead of print 4 January 2013; doi:10.1002/Jmri.23989).

\section{Acknowledgements}

We acknowledge Sarah Woods, Dr Alessia Lodi, Pia Eriksson, Galen Reed and Aaron Robinson. This work was supported by NIH UCSF Brain Tumour SPORE P50 
CA097257, NIH R01CA172845, NIH R21CA16154, NIH R01CA154915, NIH

P41EB013598, a grant from the UCSF Academic Senate and a fellowship from the American Brain Tumour Association.

\section{Author contributions}

M.M.C. designed and performed the experiments and wrote the paper. P.E.Z.L, J.J.P. and

H.A.I.Y designed and performed experiments and contributed to writing the paper.

O.M.D. performed experiments. D.B.V., S.J.N. and R.O.P. designed experiments and reviewed the paper. S.M.R. conceived the study, designed the experiments and wrote the paper.

\section{Additional information}

Supplementary Information accompanies this paper at http://www.nature.com/ naturecommunications

Competing financial interests: The authors declare no competing financial interests.

Reprints and permission information is available online at http://npg.nature.com/ reprintsandpermissions/

How to cite this article: Chaumeil, M. M. et al. Non-invasive in vivo assessment of IDH1 mutational status in glioma. Nat. Commun. 4:2429 doi: 10.1038/ncomms3429 (2013). 